\title{
Impact of Environmental Covariation in Growth and Mortality on Evolving Maturation Reaction Norms
}

\author{
Lise Marty $^{1, *}$, Ulf Dieckmann ${ }^{2}$, Marie-Joëlle Rochet $^{3}$, and Bruno Ernande ${ }^{1,2}$
}

\author{
${ }^{1}$ Laboratoire Ressources Halieutiques, Institut Français de Recherche pour l'Exploitation de la Mer (IFREMER), \\ 150 Quai Gambetta, BP 699, F-62231 Boulogne-sur-mer, France \\ ${ }^{2}$ Evolution and Ecology Program, International Institute for Applied Systems and Analysis, Schlossplatz 1, A-2361 \\ Laxenburg, Austria \\ ${ }^{3}$ Ecologie et Modèle pour l'Halieutique, IFREMER, rue de l'Ile d'Yeu, BP 21105, F-44311 Nantes Cedex 03, \\ France \\ *Corresponding author : Lise Marty, e-mail: lise.marty@ifremer.fr
}

\begin{abstract}
:
Maturation age and size have important fitness consequences through their effects on survival probabilities and body sizes. The evolution of maturation reaction norms in response to environmental covariation in growth and mortality is therefore a key subject of life-history theory. The ecoevolutionary model we present and analyze here incorporates critical features that earlier studies of evolving maturation reaction norms have often neglected: the trade-off between growth and reproduction, source-sink population structure, and population regulation through density-dependent growth and fecundity. We report the following findings. First, the evolutionarily optimal age at maturation can be decomposed into the sum of a density-dependent and a density-independent component. These components measure, respectively, the hypothetical negative age at which an individual's length would be 0 and the delay in maturation relative to this offset. Second, along any growth trajectory, individuals mature earlier when mortality is higher. This allows us to deduce, third, how the shapes of evolutionarily optimal maturation reaction norms depend on the covariation between growth and mortality (positive or negative, linear or curvilinear, and deterministic or probabilistic). Providing eco-evolutionary explanations for many alternative reaction-norm shapes, our results appear to be in good agreement with current empirical knowledge on maturation dynamics.
\end{abstract}

Keywords : phenotypic plasticity, growth-reproduction trade-off, source-sink population structure, density dependence, selection gradient. 


\section{Introduction}

Age and size at maturation have strong impacts on an individual's fitness, because they affect its reproductive potential, schedule, and efficiency (Stearns 1992; Charlesworth 1994). Maturing early increases survival until reproduction, lengthens reproductive lifespan, and reduces generation time. Maturing late increases fecundity at age, lengthens the phase of fast juvenile growth, and improves offspring survival through parental body-size effects. Furthermore, individuals face a trade-off between maturing young or at large size, since for any given growth rate earlier maturation implies smaller size.

Owing to their effects on fitness, age and size at maturation are subject to natural and/or anthropogenic selection pressures. Plastic variations in age and size at maturation are ubiquitous within species (Stearns 1992) and are often characterized by univariate reaction norms that describe either age (fig 1A) or size (fig 1B) at maturation as a function of the growth rate characterizing the experienced environmental conditions. Bivariate maturation reaction norms extend this concept to joint phenotypic plasticity in age and size at maturation (fig. 1C). Accordingly, a maturation reaction norm is the curve in the age-size plane connecting the combinations of age and size at maturation that are expressed by a given genotype for different growth rates in the age-size plane (Stearns and Crandall 1984; Stearns and Koella 1986). The evolution of maturation reaction norms has been the subject of numerous theoretical studies (e.g., Stearns and Koella 1986; Perrin and Rubin 1990; Berrigan and Koella 1994; Day and Rowe 2002; Ernande et al. 2004; Dunlop et al. 2007, 2009a, 2009b; Thériault et al. 2008; Enberg et al. 2009; Jørgensen et al. 2009). As the costs and benefits of maturing earlier or later accrue in terms of survival and/or size-dependent fecundity, the rates of somatic growth and mortality are expected to serve as primary determinants of maturation evolution. These rates are largely influenced by environmental conditions, including both biotic and abiotic factors. For instance, growth rates depend on food resources and temperature (Boggs and Ross 1993; Adolph and Porter 1993), while mortality rates are also influenced by food resources and tem- 
perature, as well as by predators and pathogens (e.g., Anholt and Werner 1995; Werner and Anholt 1996).

Rates of growth and mortality may covary positively or negatively across environmental conditions. Such covariation can have many causes. For example, positive covariation may arise from the trade-off between foraging time and predation risk: individuals that forage longer or more audaciously acquire more energy and grow faster, but at the same time are exposed to higher predation risk, and thus to higher mortality (Abrams 1991; Werner and Anholt 1993; Walters and Korman 1999). In contrast, negative covariation between growth and mortality rates may arise when the spatial distribution of food resources is heterogeneous, such that individuals in richer environmental conditions can acquire more energy than those in poorer conditions, thus benefiting both in terms of growth and survival.

Several theoretical studies have investigated the influence of covariation between growth and mortality on the evolution of maturation reaction norms. Stearns and Koella (1986) and Burd et al. (2006) analyzed different negative relationships between growth and mortality, and found various optimal reaction norms shapes: L-shaped, sigmoid, and Vshaped in Stearns and Koella (1986), curved or linear with different slopes in Burd et al. (2006). Berrigan and Koella (1994) extended the analysis to positive relationships and found other optimal shapes (flat, dome-shaped, and bowl-shaped). However, these studies used von Bertalanffy's growth model, which does not account for the crucial energy-allocation tradeoff between somatic growth and reproduction (Day and Taylor 1997). This trade-off is key to the evolution of maturation. It effectively pitches current against future reproduction: during and after maturation, energy is allocated to reproduction at the expense of somatic growth, which in turn reduces future reproduction to the extent that such reproduction increases with body size. Studies on the evolution of maturation reaction norms therefore need to account for the energy-allocation trade-off between growth and reproduction. 
Perrin and Rubin (1990) modeled growth and reproduction according to energyallocation principles and thereby obtained different optimal maturation reaction norms. However, their analysis, as those by Stearns and Koella (1986), Berrigan and Koella (1994), and Burd et al. (2006), suffered from optimizing maturation separately for each environmental condition. This approach would be appropriate only if the optimal maturation reaction norm were intended to describe combinations of age and size at maturation occurring across a large number of separately evolving populations that experience different, but constant, environmental conditions. If, in contrast, the optimal maturation reaction norm is meant to describe combinations of age and size at maturation occurring in a single evolving population whose individuals may experience a range of environmental conditions, the fitness of genotypes needs to integrate across the whole range of environmental conditions these genotypes may encounter during their lifetime (Houston and McNamara 1992; Kawecki and Stearns 1993). Considering such an aggregate measure of fitness is especially critical when environmental conditions influence offspring production. In such cases, populations exhibit source-sink dynamics, so that individuals experiencing productive conditions contribute more offspring than those experiencing unproductive conditions, which results in unequal contributions of subpopulations to the population's next generation. Several authors (Van Tienderen 1991; Houston and McNamara 1992; Kawecki and Stearns 1993; Ernande and Dieckmann 2004; Ernande et al. 2004) have proposed adequate fitness measures to model the evolution of phenotypic plasticity. So far, however, these fitness measures have not been applied to the evolution of maturation reaction norms in response to environmental covariation between growth and mortality.

A population's source-sink structure depends on the interplay between variability in the intrinsic productivity of subpopulations and the scale of population-density regulation. In classical dispersal-selection models, density regulation occurs either locally within each microenvironment, or globally in a common pool of offspring formed after reproduction in the 
microenvironments. The resultant source-sink structure gives rise to soft selection (Levene 1953) or hard selection (Dempster 1955), respectively, and is critically altered by habitat choice (Ravigné et al. 2004, 2009). Previous studies of optimal maturation reaction norms, however, did not incorporate density dependence and, more specifically, density-dependent energy acquisition, despite its important effects on somatic growth and reproductive investment, and thus on the selection pressure affecting maturation.

In this study, we investigate the evolution of maturation reaction norms under the influence of environmental covariation between growth and mortality in a manner that addresses and overcomes these three issues. We describe the trade-off between somatic growth and reproduction according to energy-allocation principles (Kozlowski and Wiegert 1986; Kozlowski 1992; Day and Taylor 1997). We use the concept of invasion fitness (Metz et al. 1992), and its application to subdivided populations (Metz and Gyllenberg 2001), to aggregate components of fitness that result from the various environmental conditions individuals may encounter. We consider populations with density regulation and source-sink structures implied by density-dependent energy acquisition, in which the growth and fecundity of individuals is affected by the population's total biomass. Combining the framework of physiologically structured population models (Metz and Diekmann 1986; De Roos et al. 1992; De Roos 1997) with a selection-gradient approach, we model the evolution of maturation reaction norms as function-valued traits (Kirkpatrick and Heckman 1989; Gomulkiewicz and Kirkpatrick 1992; Dieckmann et al. 2006; Parvinen et al. 2006).

After describing how we model life history, environmental conditions, population dynamics, and evolutionary dynamics, we analyze the influence of environmental covariation between growth potential and mortality rate on the shape of evolving maturation reaction norms. We first consider linear and nonlinear deterministic relationships between growth and mortality, and then extend our analysis to probabilistic relationships. We find that the evolutionarily optimal age at maturation involves a density-independent and a density-dependent 
component and discuss the importance of density dependence. We also show that, along any growth trajectory, individuals mature earlier when mortality is higher, and that this one simple rule helps us explain the shapes of evolving maturation reaction norms under a wide range of conditions. We finally compare our results with other theoretical and empirical studies.

\section{Model Description}

Our model describes the life history of individuals and the environmental covariation between growth and mortality underlying the population dynamics that determine the evolutionary dynamics of maturation reaction norms. Below, we present these different components in turn.

\section{Life History}

An individual's net energy acquisition rate, i.e., the surplus energy after accounting for maintenance, is assumed to scale with its weight $w$ as $w^{2 / 3}$ (Kozlowski and Wiegert 1986; Kozlowski 1992; Day and Taylor 1997). It also decreases with total population biomass $B$ because of competition for food resources, $g w^{2 / 3} /(1+\alpha B)$, where $g$ measures growth potential (or weight-specific energy acquisition) as determined by environmental condition and $1 / \alpha$ measures the population biomass at which this growth potential is halved because of density dependence. Although the allometric scaling of metabolic rates is subject to vigorous debate (e.g. Kozłowski and Konarzewski 2004; Brown et al. 2005), the qualitative results of our study remain unchanged upon varying the energy-acquisition scaling exponent over the classical range considered in bioenergetics, i.e., from $2 / 3$ to $3 / 4$. Somatic growth and fecundity compete for the allocation of surplus energy. Denoting the realized growth potential by $g_{\mathrm{r}}=g /(1+\alpha B)$, somatic growth rate and fecundity rate are then given by

$$
\frac{\partial w}{\partial a}=u g_{\mathrm{r}} w^{2 / 3}
$$

and 


$$
b(a)=(1-u) g_{\mathrm{r}} \frac{w^{2 / 3}}{w_{0}},
$$

where $a$ denotes age, $b$ fecundity rate, $w_{0}$ the weight of a newborn, and $u$ the proportion of net acquired energy devoted to somatic growth, with $1-u$ being allocated to reproduction (Kozlowski and Wiegert 1986; Kozlowski 1992; Day and Taylor 1997). During the juvenile stage, all energy is devoted to somatic growth, $u=1$, whereas during and after maturation at age $a_{\mathrm{m}}$, both functions are allocated a share of energy according to $u=\exp \left(-h\left(a-a_{\mathrm{m}}\right)\right)$, with $h$ measuring reproductive effort.

Translating weight into length according to $w=\omega l^{3}$, where $\omega$ is a constant and $l$ denotes an individual's length, we obtain the dynamics of length growth,

$$
\frac{\partial l}{\partial a}=\frac{1}{3 \omega^{1 / 3}} u g_{\mathrm{r}}
$$

At steady state, i.e., at constant total population biomass, the resultant growth trajectory is linear before maturation and afterwards converges to an asymptotic length $l_{\infty}$ (fig. 1C),

$$
l(a)= \begin{cases}l_{0}+\frac{1}{3} \frac{g_{\mathrm{r}}}{\omega^{1 / 3}} a & \text { for } a \leq a_{\mathrm{m}} \\ l\left(a_{\mathrm{m}}\right)+\frac{1}{3} \frac{g_{\mathrm{r}}}{\omega^{1 / 3} h}\left(1-e^{-h\left(a-a_{\mathrm{m}}\right)}\right) & \text { for } a>a_{\mathrm{m}},\end{cases}
$$

with length at birth $l_{0}=\left(w_{0} / \omega\right)^{1 / 3}$, length at maturation $l\left(a_{\mathrm{m}}\right)=l_{0}+a_{\mathrm{m}} g_{\mathrm{r}} /\left(3 \omega^{1 / 3}\right)$, and asymptotic length $l_{\infty}=l\left(a_{\mathrm{m}}\right)+g_{\mathrm{r}} /\left(3 h \omega^{1 / 3}\right)$. Therefore, age at maturation affects adult and asymptotic lengths, as well as size-dependent fecundity (eq. 1). When varying the reproductive effort $h$ from 0 to infinity, growth ranges from indeterminate to determinate.

Maturation responds plastically to environmental variability through its dependence on growth (Stearns and Crandall 1984; Stearns and Koella 1986). Considering only populations at steady state, a growth trajectory is characterized by its growth potential $g$. Therefore, we describe age at maturation as a function-valued trait $a_{\mathrm{m}}(\cdot)$ depending on growth potential $g$, i.e., as a univariate reaction norm that describes variation in maturation age as growth potential $g$ varies with environmental condition (fig. 1A). Length at maturation is deduced from 
$a_{\mathrm{m}}(g)$ as $l\left(a_{\mathrm{m}}(g)\right)$ (fig. 1B), and the bivariate maturation reaction norm is thus obtained as a parametric curve $\left(a_{\mathrm{m}}(g), l\left(a_{\mathrm{m}}(g)\right)\right)$ of the growth potential $g$ (fig. 1C).

For the sake of simplicity, we keep mortality rate constant throughout an individual's lifetime, although stage- or size-dependent mortality rates could be considered. An individual's survival until age $a$ is therefore obtained as

$$
s(a)=s_{0} \exp (-m a)
$$

where $s_{0}$ denotes the low survival probability affecting the earliest life stage $(a=0)$. Afterwards, the mortality rate $m$ is assumed to vary according to environmental condition. Its covariation with growth potential $g$ is described in the next section.

\section{Environmental Covariation between Growth and Mortality}

Environmental variability generates (co)variation in growth and mortality. We consider deterministic and probabilistic relationships between growth potential $g$ and mortality rate $m$. The deterministic cases allow us to examine detailed effects of the shape of the relationship on evolving maturation reaction norms, whereas probabilistic cases help us understand maturation reaction norms favored by natural selection in more realistic noisy ecological settings.

We assume that, while $g$ and $m$ are constant throughout an individual's lifetime, they vary among individuals. This can be interpreted as spatial variation in environmental conditions, or more generally as stochastic variation across microenvironments.

For deterministic relationships, mortality rate $m(g)$ is treated as a function of $g$, which is normally distributed, $g \sim N\left(\bar{g}, \sigma_{g}\right)$. To encompass both linear and nonlinear relationships, we define $m(g)$ as a parametric trade-off curve (Appendix A). Two parameters, $\beta$ and $c$, control the relationship. $\beta$ controls its slope (fig. 2A) and determines whether the two variables are independent $(\partial m(g) / \partial g=0$ for $\beta=0)$, or if they are dependent, whether they are correlated positively $(\partial m(g) / \partial g>0$ for $\beta>0)$ or negatively $(\partial m(g) / \partial g<0$ for $\beta<0) . c$ controls the curvature of the relationship (fig. 2B), which can be convex $\left(\partial^{2} m / \partial g^{2}>0\right.$ for 
$c<1$ and $\beta<0$, or for $c>1$ and $\beta>0)$, linear $(m(g)=\bar{m}+\beta(g-\bar{g})$ for $c=1)$, or concave $\left(\partial^{2} m / \partial g^{2}<0\right.$ for $c>1$ and $\beta<0$, or for $c<1$ and $\left.\beta>0\right)$.

Probabilistic covariation between growth and mortality is represented by a bivariate normal probability density function $p(g, m)$ describing the likelihood that an individual, as a result of environmental variability, experiences a specific combination of $g$ and $m$. The function $p(g, m)$ implies means $\bar{g}$ and $\bar{m}$, standard deviations $\sigma_{g}$ and $\sigma_{m}$, a slope $\beta$ of the regression of $m$ against $g$, and a correlation coefficient $\rho$ (fig. 2C). By definition, $\beta$ and $\rho$ have the same sign, so that only the correlation coefficient's absolute value $|\rho|$ conveys extra information. Linear deterministic relationships are nothing but special cases of probabilistic relationships, with $|\rho|=1$. To describe probabilistic relationships, we therefore use the same parameters as for linear deterministic relationships $\left(\bar{g}, \sigma_{g}, \bar{m}\right.$, and $\left.\beta\right)$, complemented by $|\rho|$. The standard deviation of mortality rate is $\sigma_{m}=\sigma_{g} \beta / \rho$.

\section{Population Dynamics and Evolutionary Dynamics}

We model the population dynamics resulting from life history using a physiologically structured population model (Metz and Diekmann 1986; De Roos et al. 1992; De Roos 1997). It describes the continuous-time dynamics of the density $n(a, g, m)$ of individuals aged $a$ with growth potential $g$ and mortality rate $m$ (Appendix B). Considering populations at equilibrium, an individual's length is deduced from its age and growth potential. Reproduction is panmictic and offspring distribute randomly across environmental conditions according to their frequency $p(g, m)$. The resulting gene flow among subpopulations experiencing different environmental conditions inhibits local differentiation and favors genotypes that respond plastically to environmental variability. These processes also ensure that unproductive subpopulations receive net contributions of offspring from productive ones, generating a sourcesink population structure. The stable density $\tilde{n}(a, g, m)$ of the population at equilibrium can be found analytically, up to its total biomass $\widetilde{B}$, which must be computed numerically. 
Based on this population model, we focus on the evolution of maturation reaction norms by considering the evolutionary dynamics of the function $a_{\mathrm{m}}(\cdot)$ determining age at maturation (Appendix B). We use a selection-gradient approach (Abrams 2001), which is consistent with the frameworks of both quantitative genetics (Lande 1979, 1982; Iwasa et al. 1991; Abrams et al. 1993) and adaptive dynamics (Dieckmann and Law 1996; Metz et al. 1996; Geritz et al. 1997). The selection gradient $G_{a_{\mathrm{m}}}(g)$ describes the strength and direction of selection on $a_{\mathrm{m}}(g)$ : for each $g$, a positive gradient value indicates that selection favors an increase in $a_{\mathrm{m}}(g)$, while a negative gradient value implies the opposite. The selectiongradient function $G_{a_{\mathrm{m}}}(\cdot)$ is derived from invasion fitness, following methods developed for function-valued traits (Kirkpatrick and Heckman 1989; Dieckmann et al. 2006; Parvinen et al. 2006). We use the lifetime reproductive success $R_{0}$ as a measure of invasion fitness. When density dependence regulates a population only through a single environmental variable appearing as a multiplicative factor reducing the rate of offspring production (here the inverse of total biomass, $1 / B$ reducing the fecundity $b$, eq. $1 \mathrm{~b}$ ), evolution optimizes $R_{0}$ (but not otherwise; Mylius and Diekmann 1995; Metz et al. 1996, 2008). The evolutionary dynamics of $a_{\mathrm{m}}(\cdot)$ reach a selection-induced evolutionary equilibrium when the selection gradient vanishes, $G_{a_{\mathrm{m}}^{*}}(g)=0$. Since in our model $R_{0}$ is maximized by evolution, the optimal maturation reaction norm $a_{\mathrm{m}}^{*}(\cdot)$ that cancels the selection gradient is not only convergence stable but also locally and globally evolutionarily stable (Meszéna et al. 2001; Dieckmann et al. 2006).

Throughout this study, we denote population equilibria by a tilde and evolutionary equilibria by an asterisk. We are interested in the optimal maturation reaction norms that result when both dynamics have equilibrated. 


\section{Results}

\section{Constant Growth and Mortality: Higher Mortality Favors Earlier Maturation}

To understand the basic features of maturation evolution, we first deal with the simple case of constant growth potential and mortality rate, to investigate their independent influences. In this case, maturation age and length are given by a point along a single growth trajectory (fig. 3). The evolutionarily optimal maturation age $a_{\mathrm{m}}^{*}$ can be found analytically,

$$
a_{\mathrm{m}}^{*}=\frac{-m^{2}-h m+6 h^{2}+\sqrt{m^{4}+4 h m^{3}+37 h^{2} m^{2}+60 h^{3} m+36 h^{4}}}{m^{3}+5 h m^{2}+6 h^{2} m}-l_{0} \frac{3 \omega^{1 / 3}\left(1+\alpha \tilde{B}^{*}\right)}{g}=a_{\mathrm{m}, \mathrm{i}}^{*}+\tilde{a}_{0, \mathrm{~d}}^{*},
$$

up to the total biomass $\widetilde{B}^{*}$ at population and evolutionary equilibrium, which must be determined numerically. $a_{\mathrm{m}}^{*}$ is the sum of a density-independent component $a_{\mathrm{m}, \mathrm{i}}^{*}$ and a densitydependent component $\tilde{a}_{0, \mathrm{~d}}^{*}=-l_{0} 3 \omega^{1 / 3}\left(1+\alpha \tilde{B}^{*}\right) / g$. The latter is the root of the juvenile growth function (eq. 3, first row), $l\left(\tilde{a}_{0, \mathrm{~d}}^{*}\right)=0$, and thus represents the hypothetical age at which length would equal 0 , which is negative by definition. $a_{\mathrm{m}, \mathrm{i}}^{*}=a_{\mathrm{m}}^{*}-\tilde{a}_{0, \mathrm{~d}}^{*}$ describes the density-independent timing of maturation relative to an offset measured by $\tilde{a}_{0, \mathrm{~d}}^{*}$, while $\tilde{a}_{0, \mathrm{~d}}^{*}$ itself is adjusted by density dependence. It follows that $a_{\mathrm{m}, \mathrm{i}}^{*}$ has to be positive, which is ensured as long as $h>m / 6$. In the extreme case of determinate growth, i.e., for $h \rightarrow \infty$, the optimal age at maturation relative to the age at length 0 equals twice the average individual's lifespan $1 / m, a_{\mathrm{m}}^{*}-\tilde{a}_{0, \mathrm{~d}}^{*}=a_{\mathrm{m}, \mathrm{i}}^{*} \rightarrow 2 / m$.

The density-independent component $a_{\mathrm{m}, \mathrm{i}}^{*}$ evolves towards younger ages when mortality rate $m$ increases and reproductive effort $h$ decreases, since $\partial a_{\mathrm{m}, \mathrm{i}}^{*} / \partial m<0$ and $\partial a_{\mathrm{m}, \mathrm{i}}^{*} / \partial h>0$, respectively. Notice that these changes are evolutionary and not plastic. Earlier reproduction is favored when mortality increases, because it improves an individual's likelihood to produce offspring before dying, which in turn increases its lifetime reproductive success. For reproductive effort, the evolutionary rationale relies on the trade-off between current and future reproduction. An increased reproductive effort impairs future reproduction, because it lowers growth after maturation, and thus size-dependent fecundity (eq. 1b). Concomitantly, current 
reproduction is improved, but this effect diminishes with age, since energy allocation to reproduction $1-u=1-\exp \left(-h\left(a-a_{\mathrm{m}}\right)\right)$ tends to 1 for $a \rightarrow \infty$, independent of $h>0$. The net effect is that, counter-intuitively, lifetime reproductive success decreases as reproductive effort increases, which is compensated for by maturing later and larger (eq. 3).

Mortality rate $m$ and reproductive effort $h$ have also indirect effects on the age $\tilde{a}_{0, \mathrm{~d}}^{*}$ at length 0 , through biomass $\widetilde{B}^{*}$. These effects are opposite to those on $a_{\mathrm{m}, \mathrm{i}}^{*} \cdot \tilde{a}_{0, \mathrm{~d}}^{*}$ increases when $m$ increases $\left(\partial a_{0, \mathrm{~d}}^{*} / \partial m=-\left(3 \omega^{1 / 3} l_{0} \alpha / g\right) \partial \tilde{B}^{*} / \partial m>0\right) \quad$ or $\quad h \quad$ decreases $\left(\partial a_{0, \mathrm{~d}}^{*} / \partial h=-\left(3 \omega^{1 / 3} l_{0} \alpha / g\right) \partial \tilde{B}^{*} / \partial h<0\right)$, because total biomass $\widetilde{B}^{*}$ is a decreasing function of $m\left(\partial \widetilde{B}^{*} / \partial m<0\right)$ and an increasing function of $h\left(\partial \widetilde{B}^{*} / \partial h>0\right)$ (Appendix C).

Despite these opposite effects, the net effects of mortality rate and reproductive effort on the optimal maturation age $a_{\mathrm{m}}^{*}$ are qualitatively the same as on its density-independent component $a_{\mathrm{m}, \mathrm{i}}^{*}$ (fig. 3A, 3B). However, for length at maturation, the implications of density dependence are not negligible. For higher mortality rates $m$ or lower reproductive effort $h$, the resultant decrease in total biomass $\widetilde{B}^{*}$ improves the realized growth potential $\tilde{g}_{\mathrm{r}}^{*}=g /\left(1+\alpha \tilde{B}^{*}\right)$. Despite the associated decrease in $a_{\mathrm{m}}^{*}$, the resultant length at maturation is larger than expected without this compensatory response.

Length $l_{0}$ at birth, growth potential $g$, and strength of density dependence $\alpha$ only affect age at length $0, \tilde{a}_{0, \mathrm{~d}}^{*}$. They have both a direct and an indirect effect via total biomass $\tilde{B}^{*}$. Surprisingly, these effects compensate perfectly, so that $\partial a_{0, \mathrm{~d}}^{*} / \partial l_{0}=\partial a_{0, \mathrm{~d}}^{*} / \partial g=\partial a_{0, \mathrm{~d}}^{*} / \partial \alpha=0$ (Appendix C). Therefore, $\tilde{a}_{0, \mathrm{~d}}^{*}$ and the optimal maturation age $a_{\mathrm{m}}^{*}$ are insensitive to variation in these parameters. More specifically, variation in $g$ or $\alpha$ affects $\widetilde{B}^{*}$ in such a way that the realized growth potential $\tilde{g}_{\mathrm{r}}^{*}=g /\left(1+\alpha \tilde{B}^{*}\right)$ stays constant. It follows that the optimal age and length at maturation are also left unchanged (fig. 3C). As $l_{0}$ increases, fecundity diminishes, as it is inversely related to initial weight (eq. 1b), so that $\widetilde{B}^{*}$ decreases. Changes in $\widetilde{B}^{*}$ here result in an increase in realized growth potential $\tilde{g}_{\mathrm{r}}^{*}$ that compensates for the increase in $l_{0}$ 
and results in a constant optimal age at maturation. Nevertheless, the increase in $\tilde{g}_{\mathrm{r}}^{*}$ results in a larger length at maturation (fig. 3D).

\section{Deterministic Covariation between Growth and Mortality: General Insights}

We now examine evolving maturation reaction norms under environmental covariation between growth and mortality, starting with deterministic relationships. The resulting optimal age $a_{m}^{*}(\cdot)$ at maturation has the same form as in case of constant environment, but now is a reaction norm depending on growth potential $g$,

$$
\begin{aligned}
a_{\mathrm{m}}^{*}(g) & =\frac{-m(g)^{2}-h m(g)+6 h^{2}+\sqrt{m(g)^{4}+4 h m(g)^{3}+37 h^{2} m(g)^{2}+60 h^{3} m(g)+36 h^{4}}}{m(g)^{3}+5 h m(g)^{2}+6 h^{2} m(g)}-l_{0} \frac{3 \omega^{1 / 3}\left(1+\alpha \tilde{B}^{*}\right)}{g} \\
& =a_{\mathrm{m}, \mathrm{i}}^{*}(g)+\tilde{a}_{0, \mathrm{~d}}^{*}(g)
\end{aligned}
$$

Yet, two fundamental differences to the case of constant environment exist: the densityindependent component $a_{\mathrm{m}, \mathrm{i}}^{*}(g)$ varies plastically with $g$, due to its link with mortality rate $m(g)$, and that the density-dependent component $\tilde{a}_{0, \mathrm{~d}}^{*}(g)$ also varies plastically with $g$, because total biomass $\widetilde{B}^{*}$ now stays constant whatever specific environmental condition $g$ is considered, since $\widetilde{B}^{*}$ quantifies the total population biomass across all environmental conditions. As a corollary, the evolution of age at maturation in one environmental condition depends on all other environmental conditions through their joint effect on total biomass. Moreover, owing to the source-sink structure of the population, the evolution of age at maturation can proceed for environmental conditions that would lead to non-viable populations were these considered in isolation, as offspring produced by subpopulations in viable conditions are distributed to those in non-viable conditions.

The direction of plastic changes in optimal age at maturation in response to variation in growth potential is given by the sign of the derivative of $a_{\mathrm{m}}^{*}(g)$ with respect to $g$,

$$
\frac{\partial a_{\mathrm{m}}^{*}(g)}{\partial g}=\frac{\partial m(g)}{\partial g} \frac{\partial a_{\mathrm{m}, \mathrm{i}}^{*}(g)}{\partial m(g)}+\frac{\partial \tilde{a}_{0, \mathrm{~d}}^{*}(g)}{\partial g} .
$$


Any environmental increase in mortality rate leads to a plastic decrease in the densityindependent component $a_{\mathrm{m}, \mathrm{i}}^{*}(g)$, since $\partial a_{\mathrm{m}, \mathrm{i}}^{*}(g) / \partial m(g)<0$. Plastic changes in $a_{\mathrm{m}, \mathrm{i}}^{*}(g)$ are thus opposite to the sign of growth-mortality covariation, $\partial m(g) / \partial g$, given by the parameter $\beta$ (Appendix A). Specifically, when $g$ increases, $a_{\mathrm{m}, \mathrm{i}}^{*}(g)$ plastically decreases if mortality increases with growth $(\beta>0)$, whereas it increases if mortality decreases with growth $(\beta<0)$. On the other hand, the age $\tilde{a}_{0, \mathrm{~d}}^{*}(g)$ at length 0 plastically increases with $g$ irrespective of the growth-mortality relationship, since $\partial \tilde{a}_{0, \mathrm{~d}}^{*}(g) / \partial g=3 \omega^{1 / 3} l_{0}\left(1+\alpha \tilde{B}^{*}\right) / g^{2}>0$ (notice that, in contrast to the case with constant growth and mortality, the derivative of $\tilde{B}^{*}$ is not involved, because $\tilde{B}^{*}$ depends on the entire distribution of growth potentials, rather than on any one growth potential from this distribution). Plastic changes in $\tilde{a}_{0, \mathrm{~d}}^{*}(g)$ will thus counteract those in $a_{\mathrm{m}, \mathrm{i}}^{*}(g)$ if $\beta$ is positive, and amplify them if $\beta$ is negative. In the former case, the direction of net plastic change in the optimal age at maturation $a_{\mathrm{m}}^{*}(g)$ will depend on the relative amplitude of the two components' plastic changes. Numerical results show that the plastic response of $a_{\mathrm{m}}^{*}(g)$ is qualitatively driven by $a_{\mathrm{m}, \mathrm{i}}^{*}(g)$, with $\tilde{a}_{0, \mathrm{~d}}^{*}(g)$ having only a weak effect. Consequently, fast-growing individuals mature younger than slow-growing ones if mortality rate increases with growth potential, and mature older if mortality rate decreases with growth potential.

If mortality rate and growth potential are independent $(\beta=0$, so that $\partial m(g) / \partial g=0)$, the density-independent component is fixed $\left(\partial a_{\mathrm{m}, \mathrm{i}}^{*}(g) / \partial g=0\right)$ at

$$
a_{\mathrm{m}, \mathrm{i}}^{*}(g)=\left(-m^{2}-h m+6 h^{2}+\sqrt{m^{4}+4 h m^{3}+37 h^{2} m^{2}+60 h^{3} m+36 h^{4}}\right) /\left(m^{3}+5 h m^{2}+6 h^{2} m\right) \text {. }
$$

In this case, plastic changes in the age $\tilde{a}_{0, \mathrm{~d}}^{*}(g)$ at length 0 have a more conspicuous effect. As the growth potential $g$ diminishes, $\tilde{a}_{0, \mathrm{~d}}^{*}(g)$ (which is negative) decreases, but its absolute value increases. Therefore, the optimal age at maturation is almost constant for high to moderate values of $g$, but decreases for low values of $g$. 
No analytical results are readily available to further investigate the impact of the slope and curvature of deterministic growth-mortality relationships on the shape of optimal maturation reaction norms. Therefore, in the following subsections, we combine numerical results with approximate analytical insights based on describing $a_{\mathrm{m}}^{*}(g)$ by a second-order Taylor expansion of the density-independent component $a_{\mathrm{m}, \mathrm{i}}^{*}(g)$ around the mean growth potential $\bar{g}$ (Appendix D). Comparison between the two types of results confirms that this approximation is accurate (not shown).

\section{Linear Deterministic Relationships between Growth and Mortality: Effects of Slope}

If mortality rate and growth potential are linearly related $(c=1)$ with slope $\beta$, the optimal reaction norm is approximated by

$$
a_{\mathrm{m}}^{*}(g) \approx a_{\mathrm{m}, \mathrm{i}}^{*}(\bar{g})-k_{1} \beta(g-\bar{g})+\frac{1}{2} k_{2} \beta^{2}(g-\bar{g})^{2}+\tilde{a}_{0, \mathrm{~d}}^{*}(g)
$$

where $k_{1}$ and $k_{2}$ are two positive constants that depend only on reproductive effort $h$ and mean mortality rate $\bar{m}$. This approximation enables three analytical insights. First, the position $a_{\mathrm{m}, \mathrm{i}}^{*}(\bar{g})+\tilde{a}_{0, \mathrm{~d}}^{*}(g)$ of the optimal reaction norm $a_{\mathrm{m}}^{*}(\cdot)$ is independent of the slope $\beta$, so that $\beta$ mostly affects the shape of $a_{\mathrm{m}}^{*}(\cdot)$, not its position. The constant term $a_{\mathrm{m}, \mathrm{i}}^{*}(\bar{g})$ is obviously independent of $\beta$, whereas $\tilde{a}_{0, \mathrm{~d}}^{*}(g)$ can be affected by $\beta$ through its effect on total biomass $\widetilde{B}^{*}$. Numerical results show that $\widetilde{B}^{*}$ decreases as $\beta$ increases, leading to an increase of $\tilde{a}_{0, \mathrm{~d}}^{*}(g)$ for all $g$. This is because as $\beta$ increases, highly productive environments (large $g$ ) suffer higher mortality, while less productive environments (lower $g$ ) suffer lower mortality, leading to the decrease of total biomass. However, the amplitude of this effect is generally small. Second, the linear term $-k_{1} \beta(g-\bar{g})$ confirms the result (eq. 7) that the direction of plastic changes in $a_{\mathrm{m}, \mathrm{i}}^{*}(g)$ is opposite to the sign of the slope $\beta$. Finally, the optimal age at maturation varies nonlinearly with growth potential because the quadratic term $k_{2} \beta^{2}(g-\bar{g})^{2} / 2$ is positive. Consequently, if growth potential $g$ and mortality rate $m$ are negatively correlated $(\beta<0)$, the plastic increase in $a_{\mathrm{m}, \mathrm{i}}^{*}(g)$ accelerates when $g$ increases, 
which is amplified by plastic changes in $\tilde{a}_{0, \mathrm{~d}}^{*}(g)$, whereas, when $g$ and $m$ are positively correlated $(\beta>0)$, the plastic decrease in $a_{\mathrm{m}, \mathrm{i}}^{*}(g)$ decelerates when $g$ increases, which is partly counteracted by plastic changes in $\tilde{a}_{0, \mathrm{~d}}^{*}(g)$.

Optimal maturation reaction norms rotate together with the slope $\beta$ (fig. 4). Reaction norms with positive and negative slopes are slightly convex and concave, respectively (fig. 4C-E, 4F-H; this is more visible for reaction norms in grey obtained for larger variability in growth potential). The reaction norms are curved because of the delay in age at maturation induced by the effect of decreasing growth potential on the age at length 0 , as described in the previous subsection. Two specific cases are noticeable. When mortality rate and growth potential are independent $(\beta=0)$, an almost vertical reaction norm, or maturation-age threshold, evolves (fig. 4B). In contrast, for some positive slope $\beta$ (around 0.004 in our example; fig. 4G), an almost horizontal reaction norm, or maturation-size threshold, evolves. Unlike for the maturation-age threshold, the value of $\beta$ for which such a maturation-size threshold evolves can only be assessed numerically.

Early maturation does not necessarily imply smaller length at maturation. For instance, reaction norms with negative slopes (fig. 4F), which evolve for shallow positive growthmortality relationships, generate larger lengths at maturation when maturation occurs early. This is reversed for reaction norms with positive slopes, which are favored for steeper positive growth-mortality relationships (fig. $4 \mathrm{H}$ ). Also, wider plastic variation in age and length at maturation evolves as the growth-mortality relationship becomes steeper. In this case, variability in growth potential induces a wider variation in mortality rate, which leads to the evolution of broader plastic variation in maturation age, and thus in maturation length.

Finally, the effects on the optimal maturation reaction norm of altering the degree $\sigma_{g}$ of variation in growth potential depends on the sign of the slope $\beta$. If $\beta \geq 0$, increasing $\sigma_{g}$ generates wider reaction norms, encompassing more extreme growth trajectories (fig. 4F-H). If $\beta<0$, increasing $\sigma_{g}$ also displaces reaction norms toward smaller sizes at maturation (fig. 
4C-E). In this case, productive conditions (large $g$ ) imply lower mortality, so that they make a larger contribution to $\widetilde{B}^{*}$ than unproductive conditions. Additional variability in productivity $(g)$ exacerbates this effect, so that $\widetilde{B}^{*}$ increases with $\sigma_{g}$. Consequently, for a given $g$, the realized growth potential $\tilde{g}_{\mathrm{r}}^{*}$ diminishes (compare grey and black growth trajectories for the mean growth potential, fig. $4 \mathrm{C}-\mathrm{E}$ ), while the optimal maturation age stays roughly constant. This generates a smaller size at any given maturation age.

\section{Nonlinear Deterministic Relationships between Growth and Mortality: Effects of Curvature}

We now consider nonlinear deterministic covariation between growth potential and mortality rate and examine the influence of the curvature parameter $c$ on maturation evolution. The optimal reaction norm is then approximated by

$$
a_{\mathrm{m}}^{*}(g) \approx a_{\mathrm{m}, \mathrm{i}}^{*}(\bar{g}, \beta, c)+k_{1}(\beta, c)(g-\bar{g})+\frac{1}{2} k_{2}(\beta, c)(g-\bar{g})^{2}+\tilde{a}_{0, \mathrm{~d}}^{*}(g)
$$

The main analytical insight from this approximation is that at intermediate growth potential, optimal maturation is delayed relative to the linear case for convex relationships $(c<1$ and $\beta<0$, or $c>1$ and $\beta>0)$ and accelerated for concave relationships $(c>1$ and $\beta<0$, or $c<1$ and $\beta>0$ ). The mortality rate $m$ at intermediate growth potential $g$ is indeed lower for convex relationships than for linear ones (fig. 5A, curves B and G), which favors delayed maturation, and higher for concave relationships, which favors earlier maturation (fig. 5A, curves D and E). More specifically, the constant term $a_{\mathrm{m}, \mathrm{i}}^{*}(\bar{g}, \beta, c)$ depends on both the slope $\beta$ and the curvature parameter $c$ of the relationship. It increases as $c$ increases for positive growth-mortality covariation $(\beta>0)$, whereas it decreases for negative covariation $(\beta<0)$. The effect of $c$ on the age $\tilde{a}_{0, \mathrm{~d}}^{*}(g)$ at length 0 is opposite. This is because as $c$ increases for $\beta>0$, individuals on average suffer less mortality. This results in an increase of total biomass $\widetilde{B}^{*}$, and thus in a lower age $\tilde{a}_{0, \mathrm{~d}}^{*}(g)$ at length 0 . The converse applies for $\beta<0$. However, the magnitude of the effect of $c$ on $\tilde{a}_{0, \mathrm{~d}}^{*}(g)$ is weak relative to its effect on $a_{\mathrm{m}, \mathrm{i}}^{*}(\bar{g}, \beta, c)$, so the latter dominates the effect of $c$ on the optimal maturation reaction norm $a_{\mathrm{m}}^{*}(\cdot)$. The 
coefficients $k_{1}(\beta, c)$ and $k_{2}(\beta, c)$ depend on both $\beta$ and $c$ in an analytically intractable way: no further analytical insights into their effects can be derived.

The direction of plastic changes in maturation age still depends on the sign of $\beta$ : fastgrowing individuals mature later for negative growth-mortality covariation (fig. 5B to 5D) and earlier for positive one (fig. 5E to 5G). Convex and concave reaction norms evolve for negative and positive convex growth-mortality covariation (fig. 5B, 5G), respectively, and for positive and negative concave covariation (fig. 5E, 5D), respectively. In addition, the curvature of the growth-mortality relationship exacerbates the effect of growth variability $\sigma_{g}$ relative to the linear case. As $\sigma_{g}$ increases, reaction norms are shifted toward older ages and larger sizes for convex relationships (fig. 5B, 5G), because the average mortality rate decreases (fig. 5A, grey curves B and G). The converse applies for concave relationships (fig. 5D, 5E).

\section{Probabilistic Covariation between Growth and Mortality: Effects of Correlation Coefficient}

We now focus on more realistic cases of probabilistic growth-mortality relationships and investigate the effect of the linear regression coefficient $\beta$ and of the absolute value $|\rho|$ of the correlation coefficient. Since in this case no analytical solution can be derived for the optimal age at maturation, we present only numerical results.

As in the linear deterministic case, maturation reaction norms rotate together with $\beta$, whatever the magnitude of $|\rho|$ (left to right columns in fig. 6). Earlier maturation occurs for fast growth when growth and mortality covary positively, and for slow growth when they covary negatively. Decreasing $|\rho|$, implying lower determinism in growth-mortality covariation, induces three effects (top to bottom row in fig. 6). First, optimal reaction norms shift toward older ages and larger sizes. Second, this shift is larger for growth trajectories that are subject to lower mortality rates, i.e., for steeper growth trajectories when growth and mortality are positively correlated, and for shallower growth trajectories when they are negatively correlated. Third, where this shift occurs over the nonlinear part of growth trajectories, the 
reaction norms' curvature increases, yielding concave reaction norms for positive growthmortality covariation and convex reaction norms for negative covariation.

\section{Discussion}

Optimal maturation age reflects both density-independent and density-dependent processes

A major novel result of our study is that optimal age at maturation decomposes as the sum of a density-independent and a density-dependent component. The latter represents the hypothetical negative age at which an individual's length would be zero. This means that density regulation affects optimal maturation reaction norms by shifting this offset age, with densityindependent effects acting on top of the offset. Our finding generalizes an earlier result obtained by Day and Taylor (1997), which was based on the same energy-allocation model as our study, but was restricted to non-plastic maturation, determinate growth, and densityindependent life histories (see also Lester et al. 2004). In contrast, our result holds for nonplastic and plastic maturation, determinate and indeterminate growth, and density-dependent and density-independent life histories. Below, we detail the implications of density dependence.

Density regulation in constant environments. For non-plastic maturation, density dependence results in the optimal maturation age being insensitive to growth potential, strength of density dependence, and length at birth. This new result contrasts with earlier findings by Day and Taylor (1997), who predicted a strong positive effect of growth potential on optimal maturation age, for determinate and density-independent growth.

Density regulation across a continuum of environmental conditions. We consider a continuum of environmental conditions coupled through density regulation: energy acquisition is regulated by a population's total biomass across the full range of environmental conditions. Consequently, all individuals experience the same density dependence, which, together with the random dispersal of offspring across all environmental conditions, results in a continuous 
version of hard selection (Dempster 1955; see also Ravigné et al. 2004, 2009). This coupling of subpopulations in different conditions implies a source-sink population structure, in which some subpopulations export an oversupply of offspring to others. These features have three important effects on the evolving maturation reaction norms. First, optimal maturation reaction norms are affected by the length at birth, the growth potential, and the strength of density dependence, because total biomass is independent of the population density in the specific environmental condition considered. These new results contrast with those for constant environments. Second, the source-sink structure maintains individuals under growth and mortality conditions that would cause population extinction if experienced in isolation. This enables evolution of the maturation reaction norm in these non-viable conditions. Third, the evolution of the maturation reaction norm in a given environmental condition is constrained by all other environmental conditions, since the population's total biomass equally affects the hypothetical age at length 0 across all conditions. Individuals in unproductive conditions suffer stronger density dependence than if they were isolated, because they experience a higher total biomass enabled by the more productive conditions. The evolution of a maturation reaction norm's density-dependent component is thus dominated by the productive environments. This asymmetry is exemplified by the effect of growth variation on the position and shape of maturation reaction norms (fig. 4C-E, black and grey lines). This result extends to density-dependent plastic life histories previous insights about the evolutionary effects of source-sink population structure obtained for density-dependent non-plastic (Brown and Pavlovic 1992; Holt and Gaines 1992) and density-independent plastic (Houston and McNamara 1992; Kawecki and Stearns 1993) life histories. The novelty here is that density dependence can be the mediator of the influence of productive environmental conditions on reaction-norm evolution. Previous treatments of the influence of growth and mortality on the evolution of maturation reaction norms (Stearns and Koella 1986; Perrin and Rubin 1990; Berrigan and Koella 1994; Day and 
Rowe 2002; Burd et al. 2006) missed these results, because they lacked the joint density regulation of subpopulations across different environmental conditions.

\section{One Simple Rule Helps Explain all Evolutionarily Optimal Reaction Norm Shapes}

According to general insights into life-history evolution, investing more energy into growth by delaying maturation will on average not pay off evolutionarily, if the probability of dying before reproduction is high (Stearns 1992). Increased mortality thus selects for earlier maturation. This was shown by earlier studies, which, however, were limited to non-plastic maturation (e.g., Kozłowski and Wiegert 1987). The new insight provided by our study is that this rule also applies to adaptive plasticity in age at maturation: along any growth trajectory, increased mortality selects for earlier maturation. Consequently, the shapes of evolutionarily optimal maturation reaction norms can be deduced from how growth and mortality covary across environmental conditions.

For positively correlated relationships, fast-growing individuals experience higher mortality and therefore mature earlier while whether they mature larger or smaller depends on the steepness of the relationship (fig. 4F, $4 \mathrm{H}$ and 6). Growth trajectories approach the resultant optimal maturation norms always from below. The converse holds for negatively correlated relationships except that fast-growing individuals always mature larger (fig. 4C-E and fig. 6). Considering curvilinear relationships, optimal reaction norms bulge toward younger ages for concave relationships, i.e., when mortality at intermediate growth is higher than in the linear case, and to older ages for convex ones (fig. 5). A maturation age threshold (fig. 4B) evolves when mortality is constant despite variation in growth while a maturation size threshold (fig. 4G) evolves for shallow positive linear deterministic relationships. For probabilistic relationships, lower determinism in growth-mortality covariation favors older ages and larger sizes at maturation (fig. 6), because the function $s(a, m)=s_{0} \exp (-m a)$ (which translates mortality rate $m$ into survival probability) is convex. When mortality varies around its mean $\bar{m}(g)$ on a growth trajectory with growth potential $g$, the resultant average survival therefore always 
exceeds $s(a, \bar{m}(g))$. The implied reduction in average mortality favors later maturation. This weaker average selection pressure toward early maturation relative to the linear deterministic case can be related to a secondary source-sink population structure for each growth potential: as sources experience lower mortality than sinks, they contribute more offspring to the next generation and therefore drive the evolution of maturation.

\section{Comparison with Earlier Theoretical Studies}

Effects of growth potential. Studies that describe growth using a monophasic growth model, such as von Bertalanffy's model, and fecundity as an allometric function of body size predict plastically delayed maturation when growth potential decreases (Stearns and Koella 1986; Berrigan and Koella 1994; Burd et al. 2006). This prediction is based on overlooking the energy trade-off between growth and reproduction (Day and Taylor 1997). Explicitly accounting for the underlying energy allocation, we found that, when growth varies alone, maturation is plastically delayed as growth increases, and, when growth and mortality covary, plasticity in maturation age occurs in the direction opposite to the sign of growth-mortality covariation. These results agree with previous studies based on energy-allocation principles (Perrin and Rubin 1990; Day and Rowe 2002).

Direction of reaction-norm curvature. Like several previous studies, our model predicts both concave and convex optimal maturation reaction norms. Concave reaction norms evolve for positive linear (fig. 4F-H), positive convex (fig. 5G), and negative concave (fig. 5D) deterministic growth-mortality covariation, and for positive probabilistic covariation (fig. 6). Convex reaction norms evolve for relationships with the opposite features (fig. 4C-E; 5B; 5E; 6). Also Berrigan and Koella (1994) predicted concave and convex reaction norms, respectively, for convex and concave positive deterministic growth-mortality covariation. However, for positive linear deterministic covariation, they obtained convex rather than concave reaction norms. This discrepancy with our results is again due to the use of von Bertalanffy's growth model, which favors delayed maturation as growth decreases. 
Strength of reaction-norm curvature. Using an energy-allocation model, Perrin and Rubin (1990) obtained convex and concave reaction norms for positive and negative linear deterministic covariation of growth and survival. Their results qualitatively agree with ours, although they obtained stronger curvatures. Perrin and Rubin (1990) interpreted these curvatures as resulting from the additional selective pressure toward early maturation generated by the finite lifespan they assumed in their model. Our results show that this specific assumption is not at all necessary for obtaining curved reactions norms.

\section{Model Limitations and Extensions}

The variety of shapes we have found for optimal maturation reaction norms results from the diversity of ecological settings we have considered. However, several model limitations or extensions that can be important for understanding natural maturation processes and their determinants would be interesting to explore in the future. First, following earlier studies we assumed that growth variation is purely environmental, whereas it may also be genetic. It is therefore important to realize that our results are unaffected by the nature of growth variation as long as it is independent of maturation evolution. In addition, since we were interested in the ecological determinants of maturation evolution, we did not consider genetic constraints related to the additive genetic covariance structure of the population and instead focused on evolutionary equilibria determined by vanishing selection gradients.

Second, processes modifying mortality, such as size-dependent mortality and parental effects may generate unexpected selective pressures on maturation age and size. Mortality may decrease with size, due to a lower vulnerability of larger individuals to predators, or increase with size, a typical feature of human harvest regimes (e.g., Ernande et al. 2004; Dunlop et al. 2007, 2009a, 2009b; Thériault et al. 2008; Enberg et al. 2009; Jørgensen et al. 2009; Okamoto et al. 2009). Parental effects may enhance offspring survival through better egg quality or parental care (Trippel 1995; Berkeley et al. 2004). To account for such parental effects, Stearns and Koella (1986) defined intrinsic juvenile mortality as a decreasing function 
of age at maturation. They found that increasing extrinsic juvenile mortality delayed maturation, as the higher risk of dying before reproduction due to delayed maturation was counterbalanced by the concomitant improvement of offspring survival. This conclusion was corroborated by Dunlop et al. (2007) based on an eco-evolutionary model.

Finally, density dependence might affect processes other than energy acquisition. For example the larval stage is known to be the dominant phase of density regulation in many species (Stubbs 1977; Stiling 1988; Wootton 1998). For the sake of analytical tractability, we did not include these additional processes, but they could be accommodated in future extensions.

\section{Comparison with Empirical Knowledge}

We conclude this study by highlighting the consistency between some of our results and empirical observations. For determinate growth, we predict that the optimal age at maturation is roughly proportional to twice the average individual lifespan. This result extends earlier work by Day and Taylor (1997) and Lester et al. (2004) to populations regulated through densitydependent energy acquisition. It is consistent with the empirical observation that age at maturation is approximately proportional to average adult lifespan (Charnov and Berrigan 1990; Charnov et al. 2001). Even more encouragingly, age at maturation indeed equals roughly twice the average lifespan for Clupeidae (herrings, shads, sardines, hilsa, and menhadens), Engraulidae (anchovies), Pandalidae (Pandalid shrimps), and Sander vitreus (walleye) (Charnov and Berrigan 1990). Although growth in these species is indeterminate, it declines markedly after maturation, thus approaching conditions of determinate growth.

Most empirical studies have documented maturation reaction norms with negative slopes: fast growing individuals generally mature earlier and larger, whatever the taxon (Stearns and Koella 1986; Berrigan and Koella 1994; Day and Rowe 2002). In a theoretical study, Day and Rowe (2002) showed that this pattern could arise when constant mortality is combined with a developmental size threshold. Our results show that positive growth- 
mortality covariation is an alternative, yet not mutually exclusive, explanation for negatively sloped maturation reaction norms. Indeed, an increase in mortality with improved growth agrees with most predictions from foraging theory (e.g., Werner and Anholt 1993; Walters and Korman 1999; Abrams 2001) and empirical studies within and among fish species (Beverton and Holt 1959; Pauly 1980).

Since natural growth-mortality covariation is probabilistic and tends to be positive, our results predict that dome-shaped (i.e., concave) maturation reaction norms with a negative slope will be widespread in nature, as reported by Perrin and Rubin (1990) for fish species. More recently, a number of empirical studies have estimated probabilistic maturation reaction norms (Heino et al. 2002) for numerous fish stocks: these indeed turned out to be domeshaped, or roughly linear, with negative global slopes (Grift et al. 2003, 2007; Engelhard and Heino 2004; Olsen et al. 2004, 2005; Barot et al. 2005; Mollet et al. 2007). Only very few probabilistic maturation reaction norms have been reported to exhibit positive slope (Heino et al. 2002) or to be roughly flat (Barot et al. 2004). Empirical studies for animal species other than fish appear to be scarce. Plaistow et al (2004) experimentally tested the model by Day and Rowe (2002) using soil mites and found a maturation reaction norm with negative slope. Even though in empirical studies of plant populations phenotypic plasticity of flowering onset has been extensively treated, it has mostly been examined in terms of threshold size or age for first flowering, assuming either a size-dependent (e.g., Wesselingh et al. 1997) or agedependent flowering probability (e.g., Lacey 1988). As highlighted by Burd et al. (2006), the joint phenotypic plasticity of both age and size at first flowering in plants has received little attention in empirical studies, and when both were examined together they were considered as alternatives. Consequently, empirical results in plants are not directly comparable to our theoretical predictions.

In view of an encouraging convergence between theoretical results and empirical observations, the remaining gaps in understanding all determinants of maturation reaction norms 
call for further theoretical investigations and for the empirical testing of resultant predictions. A first step towards achieving the latter goal would be to measure patterns of growthmortality covariation in wild populations together with their maturation reaction norms and to assess the observed associations in light of our theoretical predictions. Selection experiments based on controlled growth-mortality relationships would constitute an appealing alternative.

\section{Acknowledgments}

This study has been carried out with financial support from the European Commission, as part of the Specific Targeted Research Project on "Fisheries-induced evolution" (FinE, contract number SSP-2006-044276) under the Scientific Support to Policies cross-cutting activities of the European Community's Sixth Framework Programme. It does not necessarily reflect the views of the European Commission and does not anticipate the Commission's future policy in this area. UD acknowledges additional support by the European Science Foundation, the Austrian Science Fund, the Austrian Ministry of Science and Research, and the Vienna Science and Technology Fund, as well as by the European Community's Sixth Framework Programme, through the Marie Curie Research Training Network on "Fisheries-induced adaptive changes in exploited stocks" (FishACE, contract number MRTN-CT-2004-005578).

\section{APPENDIX A}

\section{Deterministic Relationships between Growth and Mortality}

We independently control the means of growth potential and mortality rate and the shape of their relationship, by defining them as sums of means and deviations, $g=\bar{g}+\Delta g$ and $m=\bar{m}+\Delta m$. We then relate $\Delta g$ and $\Delta m$ through a parametric trade-off curve with a parameter $\theta$ ranging between 0 and 1 ,

$$
\begin{aligned}
\Delta g & =\Delta g_{\text {min }}+\left(\Delta g_{\text {max }}-\Delta g_{\text {min }}\right) \theta^{1 / c}, \\
\Delta m & =\Delta m_{\text {max }}-\left(\Delta m_{\text {max }}-\Delta m_{\text {min }}\right)(1-\theta)^{1 / c},
\end{aligned}
$$


where $c$ controls the curvature of the relationship. We define $\Delta g_{\min }=-3 \sigma_{g}$ and $\Delta g_{\max }=3 \sigma_{g}$, which covers more than $99 \%$ of the total variation in $g$, and $\Delta m_{\min }=-3 \beta \sigma_{g}$ and $\Delta m_{\max }=3 \beta \sigma_{g}$, so that $\beta$ characterizes the slope between the extrema of $g$ and $m$. Rearranging equation (A1) according to these definitions, we obtain the mortality rate $m$ as a function of the growth potential $g$,

$$
m(g)=\bar{m}+3 \beta \sigma_{g}-\beta\left(\left(6 \sigma_{g}\right)^{c}-\left(g-\bar{g}+3 \sigma_{g}\right)^{c}\right)^{1 / c} .
$$

\section{APPENDIX B}

\section{Population Dynamics and Evolutionary Dynamics}

\section{Population Dynamics}

The rate of change in the density $n(a, g, m)$ of individuals aged $a$ with growth potential $g$ and mortality rate $m$ at time $t$ is given by

$$
\frac{\partial n(a, g, m)}{\partial t}=-\frac{\partial n(a, g, m)}{\partial a}-m n(a, g, m)
$$

with a boundary condition at age 0 giving the number of offspring $n(0, g, m)$ with growth potential $g$ and mortality rate $m$,

$$
n(0, g, m)=n(0) p(g, m)
$$

where $n(0)$ denotes the total number of offspring produced in the population,

$$
n(0)=\int_{m_{\min }}^{m_{\max }} \int_{g_{\min }}^{g_{\max }} \int_{a_{\mathrm{m}}(g)}^{\infty} b(a, g, B) n(a, g, m) \mathrm{d} a \mathrm{~d} g \mathrm{~d} m .
$$

Individuals randomly distribute across environmental conditions according to their frequency, as described by the probability density function $p(g, m)$ (eq. B2a). Panmictic reproduction produces a total number $n(0)$ of offspring given by the sum over all mature ages, growth potentials, and mortality rates of individual fecundities $b(a, g, B)$ (eq. 1 b) weighted by the density $n(a, g, m)$ of individuals (eq. B2b). 
Population regulation arises through competition for food resources. Density-dependent energy acquisition is based on total population biomass $B$, which is obtained as the sum over all ages, growth potentials, and mortality rates of individual weights $w(a, g)$ weighted by the density $n(a, g, m)$ of individuals,

$$
B=\int_{m_{\min }}^{m_{\max }} \int_{g_{\min }}^{g_{\max }} \int_{0}^{\infty} w(a, g) n(a, g, m) \mathrm{d} a \mathrm{~d} g \mathrm{~d} m .
$$

At steady state, $\partial \tilde{n} / \partial t=0$, equation (B1) simplifies to

$$
\frac{\partial \tilde{n}(a, g, m)}{\partial a}=-m \tilde{n}(a, g, m)
$$

which can be solved analytically to obtain the stable population density

$$
\tilde{n}(a, g, m)=\tilde{n}(0, g, m) s(a, m),
$$

where $\tilde{n}(0, g, m)=\tilde{n}(0) p(g, m)$ is the stable density of offspring after distribution across environmental conditions and $s(a, m)$ (eq. 4 ) is their survival probability until age $a$.

The population's Lotka-Euler characteristic equation is obtained by inserting equation (B5) and equation (B2a) into equation (B2b), which gives

$$
1=\int_{m_{\min }}^{m_{\max }} \int_{g_{\min }}^{g_{\max }} p(g, m) \int_{a_{\mathrm{m}}(g)}^{\infty} b(a, g, \tilde{B}) s(a, m) \mathrm{d} a \mathrm{~d} g \mathrm{~d} m .
$$

implying that at steady state, individuals will on average have one descendant, so that the population replaces itself from one generation to the next. The right-hand side of equation (B6) equals the lifetime reproductive success $R_{0}$ (Stearns 1992). Because of the dependence of fecundity $b(a, g, \widetilde{B})$ on total population biomass $\widetilde{B}$, the characteristic equation can be solved for $\widetilde{B}$. In all cases presented in this study, the characteristic equation has no analytical solution and must be solved numerically. 


\section{Evolutionary Dynamics}

According to the frameworks of quantitative genetics (QG) and adaptive dynamics (AD), the rate of evolutionary change in the average $(\mathrm{QG})$ or resident $(\mathrm{AD})$ age at maturation $a_{\mathrm{m}}(g)$ at growth rate $g$ is proportional to

$$
\frac{\mathrm{d}}{\mathrm{d} t} a_{\mathrm{m}}(g) \propto \int \sigma_{a_{\mathrm{m}}}^{2}\left(g, g^{\prime}\right) G_{a_{\mathrm{m}}}\left(g^{\prime}\right) \mathrm{d} g^{\prime}
$$

where $G_{a_{\mathrm{m}}}(\cdot)$ is the selection gradient and $\sigma_{a_{\mathrm{m}}}^{2}(\cdot, \cdot)$ is the additive genetic (QG) or mutational (AD) covariance function (e.g., Kirkpatrick and Heckman 1989; Gomulkiewicz and Kirkpatrick 1992; Ernande and Dieckmann 2004; Dieckmann et al. 2006).

$G_{a_{\mathrm{m}}}(\cdot)$ is derived from invasion fitness, which, in our study, is defined as the expected lifetime reproductive success $R_{0}\left(a_{\mathrm{m}}^{\prime}, a_{\mathrm{m}}\right)$ of a rare variant with trait $a_{\mathrm{m}}^{\prime}(\cdot)$ in a resident population with trait $a_{\mathrm{m}}(\cdot)$ that has attained its ecological attractor. This is obtained as the sum over all ages, growth potentials, and mortality rates of the product of the variant's fecundity $b_{a_{\mathrm{m}}^{\prime}(g)}\left(a, g, \tilde{B}_{a_{\mathrm{m}}}\right)$ (defined by equation $(1 \mathrm{~b})$, where $g_{\mathrm{r}}=g /\left(1+\alpha \tilde{B}_{a_{\mathrm{m}}}\right)$ with $\tilde{B}_{a_{\mathrm{m}}}$ denoting the resident's total population biomass at equilibrium, and $w=\omega l^{3}$ with $l$ following equation (3) in which $a_{\mathrm{m}}$ is replaced by $\left.a_{\mathrm{m}}^{\prime}\right)$ and its survival probability $s(a, m)$, weighted by the probability density $p(g, m)$ of offspring distribution across environmental conditions,

$$
R_{0}\left(a_{\mathrm{m}}^{\prime}, a_{\mathrm{m}}\right)=\int_{m_{\min }}^{m_{\max }} \int_{g_{\min }}^{g_{\max }} p(g, m) \int_{a_{\mathrm{m}}^{\prime}(g)}^{\infty} b_{a_{\mathrm{m}}^{\prime}(g)}\left(a, g, \tilde{B}_{a_{\mathrm{m}}}\right) s(a, m) \mathrm{d} a \mathrm{~d} g \mathrm{~d} m .
$$

$G_{a_{\mathrm{m}}}(\cdot)$ is then defined as the functional derivative of invasion fitness (Kirkpatrick and Heckman 1989; Dieckmann et al. 2006) with respect to the variant's trait $a_{\mathrm{m}}^{\prime}(\cdot)$ evaluated at the resident's trait $a_{\mathrm{m}}(\cdot)$. Whenever invasion fitness can be written as $R_{0}\left(a_{\mathrm{m}}^{\prime}, a_{\mathrm{m}}\right)=\int_{g_{\min }}^{g_{\max }} F\left(a_{\mathrm{m}}^{\prime}(g), g, \tilde{B}_{a_{\mathrm{m}}}\right) \mathrm{d} g$, this derivative is obtained (Parvinen et al. 2006) as

$$
G_{a_{\mathrm{m}}}(g)=\left.\frac{\partial}{\partial a_{\mathrm{m}}^{\prime}(g)} F\left(a_{\mathrm{m}}^{\prime}(g), g, \tilde{B}_{a_{\mathrm{m}}}\right)\right|_{a_{\mathrm{m}}^{\prime}(g)=a_{\mathrm{m}}(g)},
$$

where according to equation (B8) 


$$
F\left(a_{\mathrm{m}}^{\prime}(g), g, \tilde{B}_{a_{\mathrm{m}}}\right)=\int_{m_{\min }}^{m_{\max }} p(g, m) \int_{a_{\mathrm{m}}^{\prime}(g)}^{\infty} b_{a_{\mathrm{m}}^{\prime}(g)}\left(a, g, \tilde{B}_{a_{\mathrm{m}}}\right) s(a, m) \mathrm{d} a \mathrm{~d} m .
$$

Evolution ceases when the expected rate of evolutionary change $\mathrm{d} a_{\mathrm{m}}(g) / \mathrm{d} t$ vanishes for all values of $g$, which can happen under two conditions (Kirkpatrick and Heckman 1989; Dieckmann et al. 2006). Selection-induced evolutionary equilibria occur when the selection gradient vanishes for all values of $g, G_{a_{\mathrm{m}}^{*}}(g)=0$, so that selective forces alone are responsible for halting evolution. Covariance-induced equilibria occur when the covariance function $\sigma_{a_{\mathrm{m}}}^{2}(\cdot$,$) is singular, i.e., when \int \sigma_{a_{\mathrm{m}}}^{2}\left(g, g^{\prime}\right) G_{a_{\mathrm{m}}}\left(g^{\prime}\right) \mathrm{d} g^{\prime}=0$ for all values of $g$ while $G_{a_{\mathrm{m}}} \neq 0$. This second type of equilibrium results from constraints caused by the underlying genetic architecture. Given our limited knowledge of the genetics of maturation, $\sigma_{a_{\mathrm{m}}}^{2}(\cdot$,$) cannot be$ estimated, so this study only focuses on selection-induced equilibria.

\section{APPENDIX C}

\section{Derivatives of Total Biomass with Respect to Model Parameters}

The total biomass $\tilde{B}^{*}$ of a population at equilibrium, with optimal reaction norm $a_{m}^{*}(\cdot)$, cannot be derived analytically, whereas its derivative with respect to any model parameter $x$ can. According to equation (B6), $R_{0}\left(a_{m}^{*}, a_{m}^{*}\right)=1$ or, making explicit the dependence on total biomass, $R_{0}\left(a_{m}^{*}\left(\tilde{B}^{*}\right), \tilde{B}^{*}\right)=1$. Taking the derivative with respect to any parameter $x$ yields

$$
\begin{aligned}
& \frac{\partial}{\partial x} R_{0}\left(a_{\mathrm{m}}^{*}\left(\tilde{B}^{*}\right), \tilde{B}^{*}\right)=0 \\
& =\left.\left(\left.\frac{\partial a_{\mathrm{m}}^{*}(B)}{\partial x}\right|_{B=\tilde{B}^{*}}+\left.\frac{\partial \tilde{B}^{*}}{\partial x} \frac{\partial a_{\mathrm{m}}^{*}(B)}{\partial B}\right|_{B=\tilde{B}^{*}}\right) \frac{\partial R_{0}\left(a, \tilde{B}^{*}\right)}{\partial a}\right|_{a=a_{m}^{*}\left(\tilde{B}^{*}\right)}+\left.\frac{\partial \tilde{B}^{*}}{\partial x} \frac{\partial R_{0}\left(a_{\mathrm{m}}^{*}\left(\tilde{B}^{*}\right), B\right)}{\partial B}\right|_{B=\tilde{B}^{*}}+\left.\frac{\partial R_{0}(a, B)}{\partial x}\right|_{a=a_{\mathrm{m}}^{*}\left(\tilde{B}^{*}\right), B=\tilde{B}^{*}} .
\end{aligned}
$$

This equation can be solved for $\partial \tilde{B}^{*} / \partial x$, which yields

$$
\frac{\partial \tilde{B}^{*}}{\partial x}=-\frac{\partial a_{\mathrm{m}}^{*}(B) /\left.\partial x\right|_{B=\tilde{B}^{*}} \partial R_{0}\left(a, \tilde{B}^{*}\right) /\left.\partial a\right|_{a=a_{\mathrm{m}}^{*}\left(\tilde{B}^{*}\right)}+\partial R_{0}(a, B) /\left.\partial x\right|_{a=a_{\mathrm{m}}^{*}\left(\tilde{B}^{*}\right), B=\tilde{B}^{*}}}{\partial a_{\mathrm{m}}^{*}(B) /\left.\partial B\right|_{B=\tilde{B}^{*}} \partial R_{0}\left(a, \tilde{B}^{*}\right) /\left.\partial a\right|_{a=a_{\mathrm{m}}^{*}\left(\tilde{B}^{*}\right)}+\partial R_{0}\left(a_{\mathrm{m}}^{*}\left(\tilde{B}^{*}\right), B\right) /\left.\partial B\right|_{B=\tilde{B}^{*}}} .
$$


Applying this method to mortality rate $m$ and reproductive investment $h$, we can show that $\partial \tilde{B}^{*} / \partial m>0$ and $\partial \tilde{B}^{*} / \partial h<0$ when $w_{0}=\omega l_{0}^{3}<\left(\tilde{g}_{\mathrm{r}}^{*} / m\right)^{3}$ or, equivalently, when $l_{0}<\tilde{g}_{\mathrm{r}}^{*} /\left(m \omega^{1 / 3}\right)$, i.e., when the length of a newborn is smaller than three times the juvenile growth rate $\tilde{g}_{\mathrm{r}}^{*} /\left(3 \omega^{1 / 3}\right)$ divided by the mortality rate $m$, a condition that holds for almost any realistic population.

Applying the same method to growth potential $g$, strength $\alpha$ of density dependence, and length $l_{0}$ at birth, we obtain

$$
\frac{\partial \widetilde{B}^{*}}{\partial g}=\frac{1+\alpha \widetilde{B}^{*}}{g \alpha}, \frac{\partial \tilde{B}^{*}}{\partial \alpha}=-\frac{\tilde{B}^{*}}{\alpha}, \text { and } \frac{\partial \widetilde{B}^{*}}{\partial l_{0}}=-\frac{1+\alpha \widetilde{B}^{*}}{l_{0} \alpha} .
$$

The three derivatives of $\tilde{a}_{0, \mathrm{~d}}^{*}$ with respect to these parameters are thus equal to zero:

$$
\begin{aligned}
& \partial a_{0, \mathrm{~d}}^{*} / \partial g=3 \omega^{1 / 3} l_{0}\left[\left(1+\alpha \tilde{B}^{*}\right) / g-\alpha \partial \tilde{B}^{*} / \partial g\right] / g=0, \\
& \partial a_{0, \mathrm{~d}}^{*} / \partial \alpha=-3 \omega^{1 / 3} l_{0}\left(\tilde{B}^{*}+\alpha \partial \tilde{B}^{*} / \partial \alpha\right] / g=0, \\
& \partial a_{0, \mathrm{~d}}^{*} / \partial l_{0}=-3 \omega^{1 / 3}\left[\left(1+\alpha \tilde{B}^{*}\right)+l_{0} \alpha \partial \tilde{B}^{*} / \partial l_{0}\right] / g=0 .
\end{aligned}
$$

\section{APPENDIX D}

\section{Approximations for Deterministic Growth-Mortality Relationships}

The optimal maturation reaction norm $a_{\mathrm{m}}^{*}(g)$ can be approximated by a second-order Taylor expansion of its density-independent component $a_{\mathrm{m}, \mathrm{i}}^{*}(g)$ around mean growth potential $\bar{g}$,

$$
a_{\mathrm{m}}^{*}(g) \approx a_{\mathrm{m}, \mathrm{i}}^{*}(\bar{g})+\left.\frac{\partial a_{\mathrm{m}, \mathrm{i}}^{*}(g)}{\partial g}\right|_{g=\bar{g}}(g-\bar{g})+\left.\frac{1}{2} \frac{\partial^{2} a_{\mathrm{m}, \mathrm{i}}^{*}(g)}{\partial g^{2}}\right|_{g=\bar{g}}(g-\bar{g})^{2}+\tilde{a}_{0, \mathrm{~d}}^{*}(g),
$$

where the constant term $a_{\mathrm{m}, \mathrm{i}}^{*}(\bar{g})$ is the density-independent component at mean growth potential, the coefficient of the first-order term describes the linear effect on $a_{\mathrm{m}, \mathrm{i}}^{*}(g)$ of variation in growth potential $g$ around its mean $\bar{g}$, and the coefficient of the second-order term describes the corresponding quadratic effect. 


\section{Literature Cited}

Abrams, P. A. 1991. Life history and the relationship between food availability and foraging effort. Ecology 72:1242-1252.

- 2001. Modelling the adaptive dynamics of traits involved in inter- and intraspecific interactions: An assessment of three methods. Ecology Letters 4:166-175.

Abrams, P. A., H. Matsuda, and Y. Harada. 1993. Evolutionarily unstable fitness maxima and stable fitness minima of continuous traits. Evolutionary Ecology 7:465-487.

Adolph, S. C., and W. P. Porter. 1993. Temperature, activity, and lizard life histories. The American Naturalist 142:273-295.

Anholt, B. R., and E. E. Werner. 1995. Interaction between food availability and predation mortality mediated by adaptive behavior. Ecology 76:2230-2234.

Barot, S., M. Heino, M. J. Morgan, and U. Dieckmann. 2005. Maturation of Newfoundland American plaice (Hippoglossoides platessoides): long-term trends in maturation reaction norms despite low fishing mortality? ICES Journal of Marine Science 62:56-64.

Barot, S., M. Heino, L. O'Brien, and U. Dieckmann. 2004. Long-term trend in the maturation reaction norm of two cod stocks. Ecological Applications 14:1257-1271.

Berkeley, S. A., C. Chapman, and S. M. Sogard. 2004. Maternal age as a determinant of larval growth and survival in a marine fish, Sebastes melanops. Ecology 85:1258-1264.

Berrigan, D., and J. Koella. 1994. The evolution of reaction norms: simple models for age and size at maturity. Journal of Evolutionary Biology 7:549-566.

Beverton, R. J. H., and S. J. Holt. 1959. A review of the lifespans and mortality rates of fish in nature, and their relation to growth and other physiological characteristics. Ciba Foundation Symposium on Ageing 5:142-177.

Boggs, C. L., and C. L. Ross. 1993. The effect of adult food limitation on life history traits in Speyeria mormonia (Lepidoptera: Nymphalidae). Ecology 74:433-441. 
Brown, J. H., G. B. West, and B. J. Enquist. 2005. Yes, West, Brown and Enquist"s model of allometric scaling is both mathematically correct and biologically relevant. Functional Ecology 19:735-738.

Brown, J., and N. Pavlovic. 1992. Evolution in heterogeneous environments: Effects of migration on habitat specialization. Evolutionary Ecology 6:360-382.

Burd, M., J. Read, G. D. Sanson, and T. Jaffré . 2006. Age-size plasticity for reproduction in monocarpic plants. Ecology 87:2755-2764.

Charlesworth, B. 1994. Evolution in age-structured populations. 2nd ed. Cambridge University Press, Cambridge.

Charnov, E. L., and D. Berrigan. 1990. Dimensionless numbers and life history evolution: age of maturity versus the adult lifespan. Evolutionary Ecology 4:273-275.

Charnov, E. L., T. F. Turner, and K. O. Winemiller. 2001. Reproductive constraints and the evolution of life histories with indeterminate growth. Proceedings of the National Academy of Sciences of the United States of America 98:9460-9464.

Day, T., and L. Rowe. 2002. Developmental thresholds and the evolution of reaction norms for age and size at life-history transitions. American Naturalist 159:338-350.

Day, T., and P. D. Taylor. 1997. Von Bertalanffy's growth equation should not be used to model age and size at maturity. American Naturalist 149:381-393.

De Roos, A. M. 1997. A gentle introduction to physiologically structured population models. Pages 119-204 in S. Tuljapurkar and H. Caswell, eds. Structured Population Models in Marine, Terrestrial and Freshwater Systems. Chapman-Hall, New York.

De Roos, A. M., O. Diekmann, and J. A. J. Metz. 1992. Studying the dynamics of structured population models: a versatile technique and its application to Daphnia. American Naturalist 139:123-147. 
Dempster, E. R. 1955. Maintenance of heterogeneity. Cold Spring Harbor Symposium on Quantitative Biology 20:25-32.

Dieckmann, U., M. Heino, and K. Parvinen. 2006. The adaptive dynamics of function-valued traits. Journal of Theoretical Biology 241:370-389.

Dieckmann, U., and R. Law. 1996. The dynamical theory of coevolution: a derivation from stochastic ecological processes. Journal of Mathematical Biology 34:579-612.

Dunlop, E. S., M. L. Baskett, M. Heino, and U. Dieckmann. 2009a. Propensity of marine reserves to reduce the evolutionary effects of fishing in a migratory species. Evolutionary Applications 2:371-393.

Dunlop, E. S., M. Heino, and U. Dieckmann. 2009b. Eco-genetic modeling of contemporary life-history evolution. Ecological Applications 19:1815-1834.

Dunlop, E. S., B. J. Shuter, M. Heino, and U. Dieckmann. 2007. The demographic and evolutionary consequences of selective mortality: predictions from an eco-genetic model for smallmouth bass. Transactions of the American Fisheries Society 136:749-765.

Enberg, K., C. Jørgensen, E. S. Dunlop, M. Heino, and U. Dieckmann. 2009. Implications of fisheries-induced evolution for stock rebuilding and recovery. Evolutionary Applications 2:394-414.

Engelhard, G. H., and M. Heino. 2004. Maturity changes in Norwegian spring-spawning herring Clupea harengus: compensatory or evolutionary responses? Marine Ecology Progress Series 272:245-256.

Ernande, B., and U. Dieckmann. 2004. The evolution of phenotypic plasticity in spatially structured environments: implications of intraspecific competition, plasticity costs and environmental characteristics. Journal of Evolutionary Biology 17:613-628. 
Ernande, B., U. Dieckmann, and M. Heino. 2004. Adaptive changes in harvested populations: plasticity and evolution of age and size at maturation. Proceedings of the Royal Society B: Biological Sciences 271:415-423.

Geritz, S. A. H., É. Kisdi, G. Meszéna, and J. A. J. Metz. 1997. Evolutionarily singular strategies and the adaptive growth and branching of the evolutionary tree. Evolutionary Ecology 12:35-57.

Gomulkiewicz, R., and M. Kirkpatrick. 1992. Quantitative genetics and the evolution of reaction norms. Evolution 46:390-411.

Grift, R. E., M. Heino, A. D. Rijnsdorp, S. B. M. Kraak, and U. Dieckmann. 2007. Threedimensional maturation reaction norms for North Sea plaice. Marine Ecology Progress Series 334:213-224.

Grift, R. E., A. D. Rijnsdorp, S. Barot, M. Heino, and U. Dieckmann. 2003. Fisheries-induced trends in reaction norms for maturation in North Sea plaice. Marine Ecology Progress Series 257:247-257.

Heino, M., U. Dieckmann, and O. R. Godø. 2002. Estimating reaction norms for age and size at maturation with reconstructed immature size distributions: a new technique illustrated by application to Northeast Arctic cod. ICES Journal of Marine Science 59:562575.

Holt, R., and M. Gaines. 1992. Analysis of adaptation in heterogeneous landscapes: Implications for the evolution of fundamental niches. Evolutionary Ecology 6:433-447.

Houston, A., and J. McNamara. 1992. Phenotypic plasticity as a state-dependent life-history decision. Evolutionary Ecology 6:243-253.

Iwasa, I., A. Pomianski, and S. Nee. 1991. The evolution of costly mate preferences. II, The handicap principle. Evolution 45:1431-1442. 
Jørgensen, C., B. Ernande, and Ø. Fiksen. 2009. Size-selective fishing gear and life history evolution in the Northeast Arctic cod. Evolutionary Applications 2:356-370.

Kawecki, T. J., and S. C. Stearns. 1993. The evolution of life histories in spatially heterogeneous environments: optimal reaction norms revisited. Evolutionary Ecology 7:155174.

Kirkpatrick, M., and N. Heckman. 1989. A quantitative genetic model for growth, shape, reaction norms, and other infinite-dimensional characters. Journal of Mathematical Biology 27:429-450.

Kozłowski, J., and M. Konarzewski. 2004. Is West, Brown and Enquist's Model of Allometric Scaling Mathematically Correct and Biologically Relevant? Functional Ecology 18:283-289.

Kozlowski, J. 1992. Optimal allocation of resources to growth and reproduction: Implications for age and size at maturity. Trends in Ecology \& Evolution 7:15-19.

Kozlowski, J., and R. G. Wiegert. 1986. Optimal allocation of energy to growth and reproduction. Theoretical Population Biology 29:16-37.

Kozłowski, J., and R. G. Wiegert. 1987. Optimal age and size at maturity in annuals and perennials with determinate growth. Evolutionary Ecology 1:231-244.

Lacey, E. P. 1988. Latitudinal variation in reproductive timing of a short-lived monocarp, Daucus carota (Apiaceae). Ecology 69:220-232.

Lande, R. 1979. Quantitative genetic analysis of multivariate evolution, applied to brain: body size allometry. Evolution 33:402-416.

—. 1982. Quantitative genetic theory of life history evolution. Ecology 63:607-615.

Lester, N. P., B. J. Shuter, and P. A. Abrams. 2004. Interpreting the von Bertalanffy model of somatic growth in fishes: the cost of reproduction. Proceedings of the Royal Society B: Biological Sciences 271:1548. 
Levene, H. 1953. Genetic equilibrium when more than one ecological niche is available. American Naturalist 87:331.

Meszéna, G., É. Kisdi, U. Dieckmann, S. A. H. Geritz, and J. A. J. Metz. 2001. Evolutionary optimisation models and matrix games in the unified perspective of adaptive dynamics. Selection 2:1585-1931.

Metz, J. A. J., and O. Diekmann. 1986. The dynamics of physiologically structured populations. Springer-Verlag, Berlin.

Metz, J. A. J., and M. Gyllenberg. 2001. How should we define fitness in structured metapopulation models? Including an application to the calculation of evolutionarily stable dispersal strategies. Proceedings of the Royal Society B: Biological Sciences 268:499508.

Metz, J. A. J., S. D. Mylius, and O. Diekmann. 1996. When does evolution optimise? On the relation between types of density dependence and evolutionarily stable life history parameters. IIASA Working Paper WP-96-004. available online at www.iiasa.ac.at/cgibin/pubsrch?WP96004.

—. 2008. When does evolution optimize? Evolutionary Ecology Research 10:629-654.

Metz, J. A. J., R. M. Nisbet, and S. A. H. Geritz. 1992. How should we define "fitness" for general ecological scenarios? Trends in Ecology \& Evolution 7:198-202.

Mollet, F. M., S. B. M. Kraak, and A. D. Rijnsdorp. 2007. Fisheries-induced evolutionary changes in maturation reaction norms in North Sea sole Solea solea. Marine Ecology Progress Series 351:189-199.

Mylius, S. D., and O. Diekmann. 1995. On evolutionarily stable life histories, optimization and the need to be specific about density dependence. Oikos 74:218-224. 
Okamoto, K. W., R. Whitlock, P. Magnan, and U. Dieckmann. 2009. Mitigating fisheriesinduced evolution in lacustrine brook charr (Salvelinus fontinalis) in southern Quebec, Canada. Evolutionary Applications 2:415-437.

Olsen, E. M., M. Heino, G. R. Lilly, M. J. Morgan, J. Brattey, B. Ernande, and U. Dieckmann. 2004. Maturation trends indicative of rapid evolution preceded the collapse of northern cod. Nature 428:932-935.

Olsen, E. M., G. R. Lilly, M. Heino, J. B. Morgan, J. Brattey, and U. Dieckmann. 2005. Assessing changes in age and size at maturation in collapsing populations of Atlantic cod (Gadus morhua). Canadian Journal of Fisheries and Aquatic Sciences 62:811-823.

Parvinen, K., U. Dieckmann, and M. Heino. 2006. Function-valued adaptive dynamics and the calculus of variations. Journal of Mathematical Biology 52:1-26.

Pauly, D. 1980. On the interrelationships between natural mortality, growth parameters, and mean environmental temperature in 175 fish stocks. ICES Journal of Marine Science 39:175-192.

Perrin, N., and J. F. Rubin. 1990. On dome-shaped norms of reaction for size-to-age at maturity in fishes. Functional Ecology 4:53-57.

Plaistow, S. J., C. T. Lapsley, A. P. Beckerman, and T. G. Benton. 2004. Age and size at maturity: sex, environmental variability and developmental thresholds. Proceedings of the Royal Society B: Biological Sciences 271:919-924.

Ravigné, V., U. Dieckmann, and I. Olivieri. 2009. Live where you thrive: joint evolution of habitat choice and local adaptation facilitates specialization and promotes diversity. American Naturalist 174:141-169.

Ravigné, V., I. Olivieri, and U. Dieckmann. 2004. Implications of habitat choice for protected polymorphisms. Evolutionary Ecology Research 6:125-145.

Stearns, S. C. 1992. The Evolution of Life Histories. Oxford University Press, Oxford. 
Stearns, S. C., and R. E. Crandall. 1984. Plasticity for age and size at sexual maturity: a lifehistory response to unavoidable stress. Pages 13-33 in G. Potts and R. J. Wootton, eds. Fish Reproduction. Academic Press, London.

Stearns, S. C., and J. C. Koella. 1986. The evolution of phenotypic plasticity in life-history traits: predictions of reaction norms for age and size at maturity. Evolution 40:893913.

Stiling, P. 1988. Density-dependent processes and key factors in insect populations. Journal of Animal Ecology 57:581-593.

Stubbs, M. 1977. Density dependence in the life-cycles of animals and its importance in Kand r-strategies. Journal of Animal Ecology 46:677-688.

Thériault, V., E. S. Dunlop, U. Dieckmann, L. Bernatchez, and J. J. Dodson. 2008. The impact of fishing-induced mortality on the evolution of alternative life-history tactics in brook charr. Evolutionary Applications 1:409-423.

Trippel, E. A. 1995. Age at maturity as a stress indicator in fisheries. BioScience 45:759-771.

Van Tienderen, P. H. 1991. Evolution of generalists and specialist in spatially heterogeneous environments. Evolution 45:1317-1331.

Walters, C., and J. Korman. 1999. Linking recruitment to trophic factors: revisiting the Beverton-Holt recruitment model from a life history and multispecies perspective. Reviews in Fish Biology and Fisheries 9:187-202.

Werner, E. E., and B. R. Anholt. 1993. Ecological consequences of the trade-off between growth and mortality rates mediated by foraging activity. American Naturalist $142: 242-272$.

Werner, E. E., and B. R. Anholt. 1996. Predator-induced behavioral indirect effects: consequences to competitive interactions in Anuran larvae. Ecology 77:157-169. 
Wesselingh, R. A., D. T. J. Jong, P. G. Klinkhamer, and L. A. Boorman. 1997. Threshold size for flowering in different habitats: effects of size-dependant growth and survival. Ecology 78:2118-2132.

Wootton, R. J. 1998. Ecology of Teleost Fishes. 2nd ed. Kluwer Academic Publishers, London.

\section{Figure Captions}

Figure 1: Schematic illustration of maturation reaction norms. A, Univariate maturation reaction norm $a_{\mathrm{m}}(\cdot)$, showing the relationship between maturation age $a_{\mathrm{m}}(g)$ and growth potential $g$. The range of growth potentials, $g_{\min }=\bar{g}-3 \sigma_{g}$ to $g_{\max }=\bar{g}+3 \sigma_{g}$, with a mean growth potential $\bar{g}$ and a standard deviation $\sigma_{g}$, characterizes the extent of heterogeneity in the environmental conditions individuals may encounter. $B$, Resulting univariate maturation reaction norm $l_{\mathrm{m}}($.$) , showing the relationship between maturation length l_{\mathrm{m}}(g)$ and growth potential $g$, deduced from the maturation age $a_{\mathrm{m}}(g)$ and the growth potential $g$ using the growth trajectory (eq. 3). $C$, Bivariate maturation reaction norm $\left(a_{\mathrm{m}}(\cdot), l\left(a_{\mathrm{m}}(\cdot)\right)\right)$, showing the combinations of maturation age $a_{\mathrm{m}}(g)$ and maturation length $l\left(a_{\mathrm{m}}(g)\right.$ that result from different growth potentials $g$.

Figure 2: Examples of covariation between growth potential and mortality rate. A, Linear deterministic relationships $(c=1)$ between $g$ and $m$ for slopes $\beta=-0.005,-0.004,-0.002$, $0,0.002,0.004,0.005 \mathrm{~g}^{-1 / 3} . B$, Nonlinear deterministic relationships between $g$ and $m$ for curvature parameters $c=0.5,1,2.5$ and slopes $\beta=-0.002,0,0.002 \mathrm{~g}^{-1 / 3} . C$, Probabilistic relationship between $g$ and $m$. Thin lines show equally spaced isoprobability levels of the probability density function $p(g, m)$ for a regression slope $\beta=0.002 \mathrm{~g}^{-1 / 3}$ and a correlation coefficient $\rho=0.5$. The thick line shows the linear regression of mortality rate $m$ on growth potential $g$. Other parameters: $\bar{m}=0.2 \mathrm{yr}^{-1}, \bar{g}=45 \mathrm{~g}^{1 / 3} \cdot \mathrm{yr}^{-1}$, and $\sigma_{g}=5 \mathrm{~g}^{1 / 3} \cdot \mathrm{yr}^{-1}$.

Figure 3: Evolutionarily optimal age and length at maturation for constant growth potential 
and mortality rate. Growth trajectories and optimal combinations of maturation age and length for different values of the following parameters: $A$, mortality rates $m=0.1,0.2,0.3,0.4,0.5$ $\mathrm{yr}^{-1} ; B$, reproductive efforts $h=0.1,0.2,0.4,0.8,1.6 \mathrm{yr}^{-1} ; C$, growth potentials $g=20,30$, $40,50,60,70 \mathrm{~g}^{1 / 3} \cdot \mathrm{yr}^{-1}$ and strengths of density dependence, $\alpha=10^{-14}, 10^{-13}, 10^{-12}, 10^{-11}, 10^{-}$ ${ }^{10} \mathrm{~g}^{-1} ; D$, weights $w_{0}$ at birth, and thus lengths at birth $l_{0}=\left(w_{0} / \omega\right)^{1 / 3}$, with $w_{0}=0.1,0.2,0.3$, 0.4, $0.5 \mathrm{~g}$. Other parameters (unless stated otherwise): $s_{0}=20.4210^{-6}, \omega=0.0104 \mathrm{~g} \cdot \mathrm{cm}^{3}$, $\alpha=8.68510^{-12} \mathrm{~g}^{-1}, h=0.5 \mathrm{yr}^{-1}, w_{0}=0.364 \mathrm{~g}, m=0.2 \mathrm{yr}^{-1}$, and $g=45 \mathrm{~g}^{1 / 3} \cdot \mathrm{yr}^{-1}$. These choices are meant to represent the life history of a long-lived fish such as cod (Gadus morhua), but the numerical values do not affect any qualitative findings.

Figure 4: Evolutionarily optimal maturation reaction norms for linear deterministic relationships $(c=1)$ between growth and mortality. A, Growth-mortality relationships for different slopes $\beta=-0.005(C),-0.004(D),-0.002(E), 0(B), 0.002(E), 0.004(F), 0.005(G) \mathrm{g}^{-1 / 3}$, and for two standard deviations of growth potential $\sigma_{g}=5$ (black lines), 10 (grey lines) $\mathrm{g}^{1 / 3} \cdot \mathrm{yr}^{-1} . B$ to $H$, Resulting optimal maturation reaction norms (thick lines) and realized growth trajectories (thin lines, corresponding to minimum, mean, and maximum growth potentials, $g_{\text {min }}=\bar{g}-3 \sigma_{g}, \bar{g}$, and $g_{\max }=\bar{g}+3 \sigma_{g}$ ). Other parameters as in figures 2 and 3.

Figure 5: Evolutionarily optimal maturation reaction norms for nonlinear deterministic relationships between growth and mortality. A, Growth-mortality relationship for different combinations of slope $\beta$ and curvature parameter $c,(\beta, c)=\left(-0.002 \mathrm{~g}^{-1 / 3}, 0.5\right)(B),(-0.002$ $\left.\mathrm{g}^{-1 / 3}, 1\right)(C),\left(-0.002 \mathrm{~g}^{-1 / 3}, 2.5\right)(D),\left(0.002 \mathrm{~g}^{-1 / 3}, 2.5\right)(E),\left(0.002 \mathrm{~g}^{-1 / 3}, 1\right)(F),\left(0.002 \mathrm{~g}^{-1 / 3}\right.$, 0.5) $(G)$, and for two standard deviations of growth potential $\sigma_{g}=5$ (black lines), 10 (grey lines) $\mathrm{g}^{1 / 3} \cdot \mathrm{yr}^{-1} \cdot B$ to $G$, Resulting optimal maturation reaction norms (thick lines) and realized growth trajectories (thin lines, corresponding to minimum, mean, and maximum growth potentials, $g_{\min }=\bar{g}-3 \sigma_{g}, \bar{g}$, and $g_{\max }=\bar{g}+3 \sigma_{g}$ ). Other parameters as in figures 2 and 3.

Figure 6: Evolutionarily optimal maturation reaction norms for probabilistic relationships 
between growth and mortality. Optimal maturation reaction norms (thick lines) and realized growth trajectories (thin lines, corresponding to minimum, mean, and maximum growth potentials, $g_{\min }=\bar{g}-3 \sigma_{g}, \bar{g}$, and $g_{\max }=\bar{g}+3 \sigma_{g}$ ) resulting for probabilistic growth-mortality relationships with different combinations of regression slope $\beta$ and correlation coefficient $\rho$, and for two standard deviations of growth potential $\sigma_{g}=5$ (black lines), 10 (grey lines) $\mathrm{g}^{1 / 3} \cdot \mathrm{yr}^{-1}$. Other parameters as in figures 2 and 3 . 

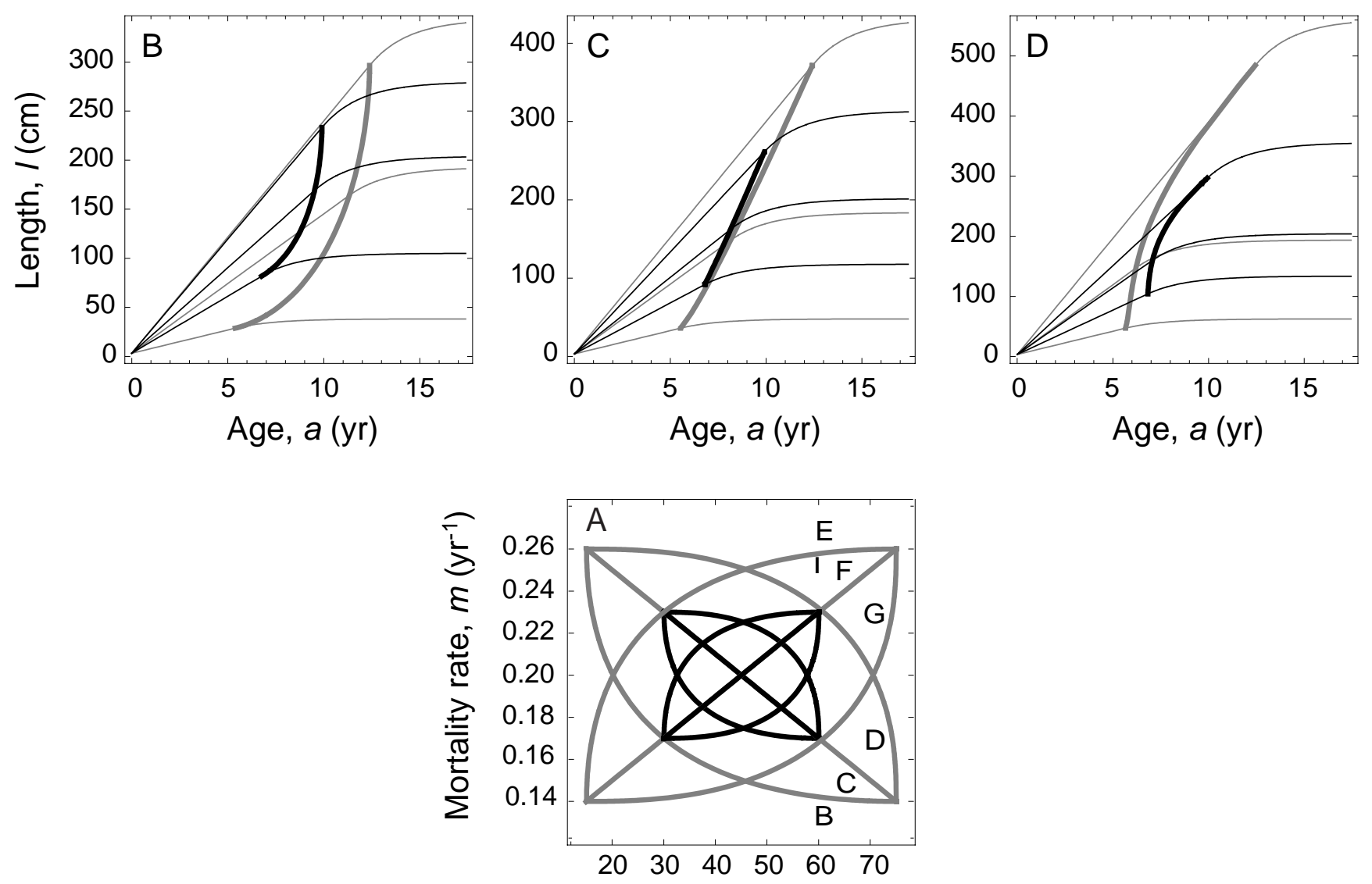

Growth potential, $g\left(g^{1 / 3} \mathrm{yr}^{-1}\right)$
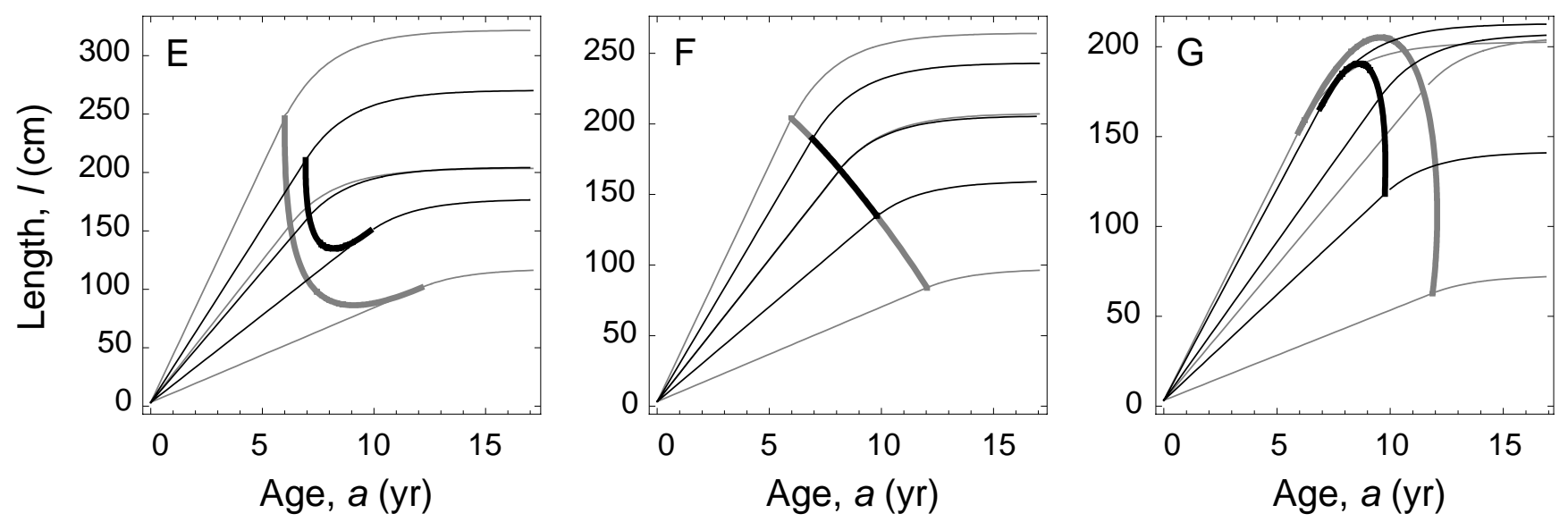

Figure 5. Marty et al. 


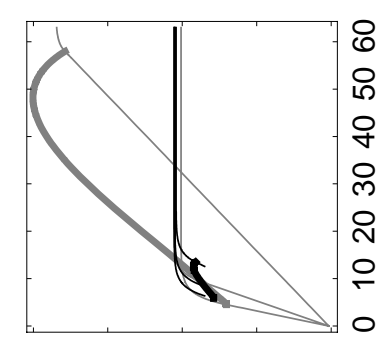

욱윰 웡ㅇํㅇ
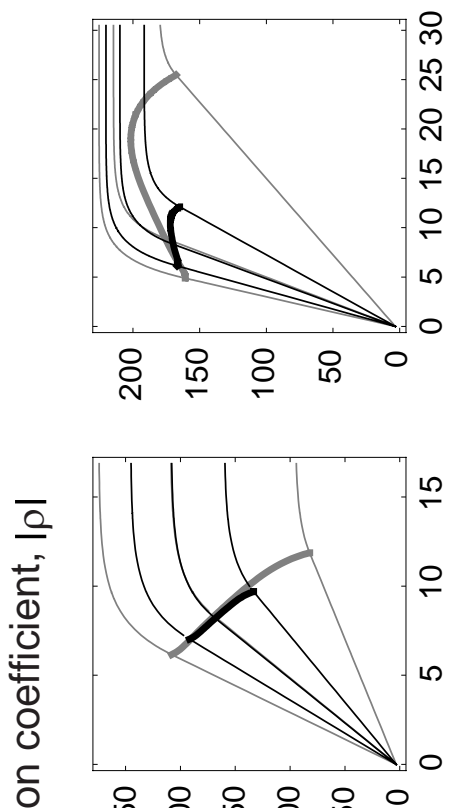

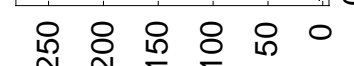

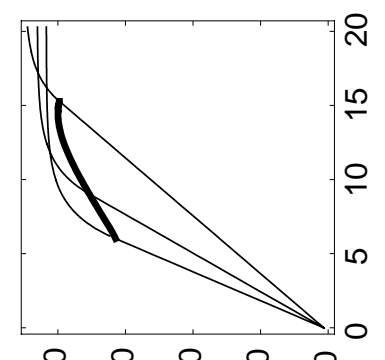

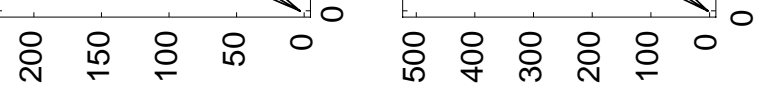

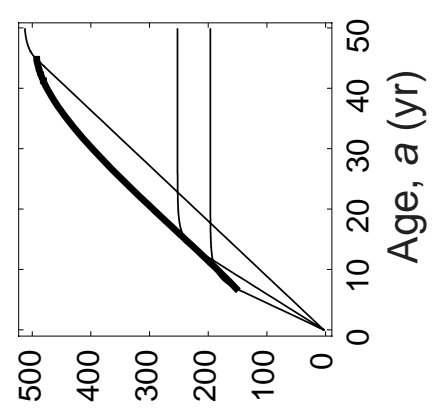

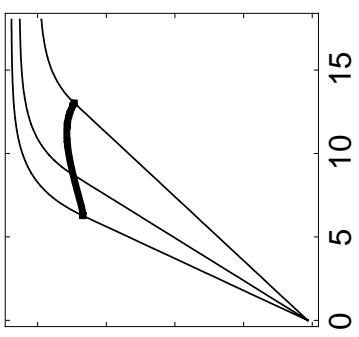

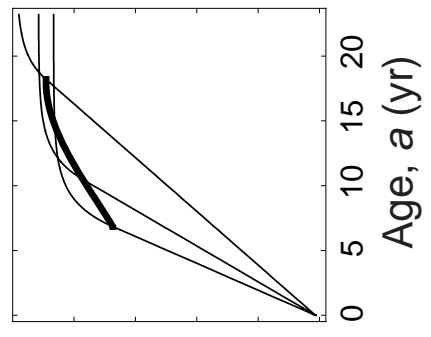

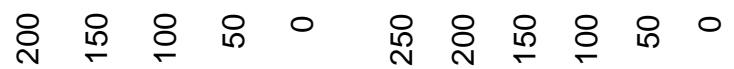
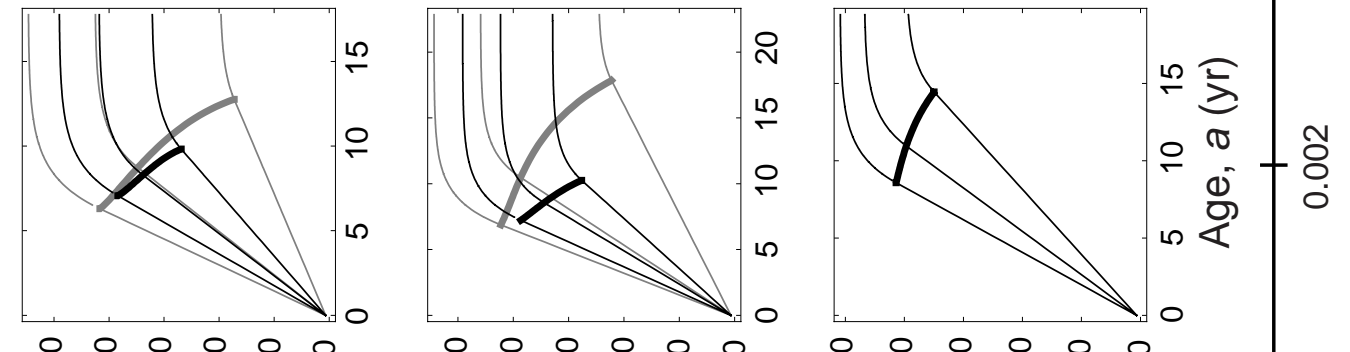

(0)
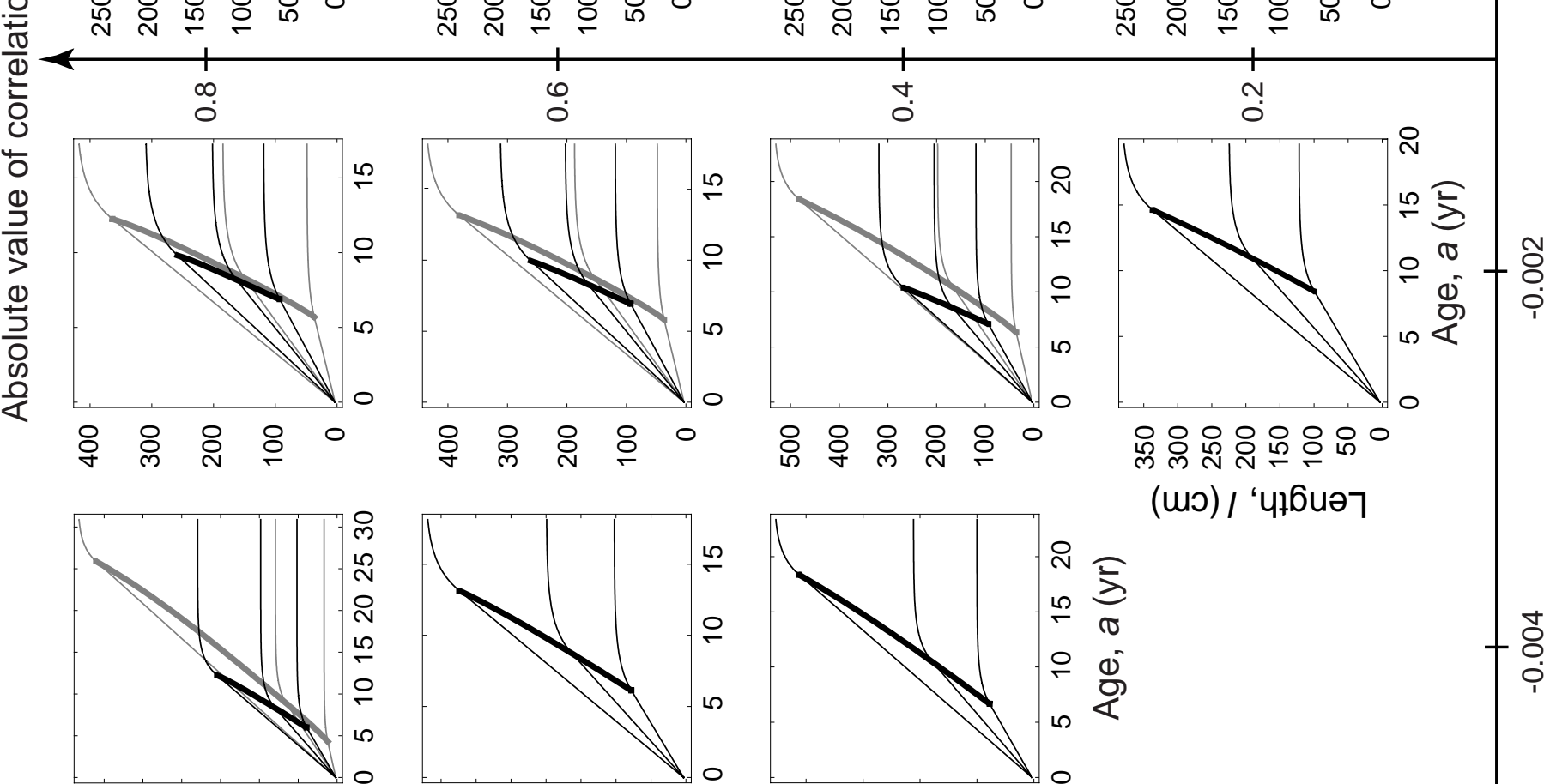

\& \& \&

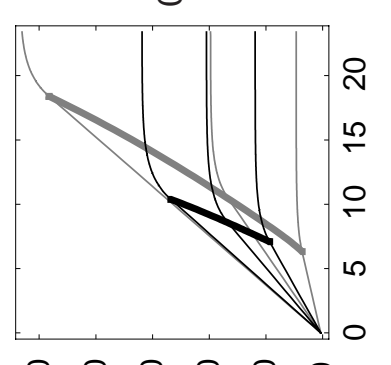

৪ ㅇํㅇ \&

(u०) / 'บ1бuәา

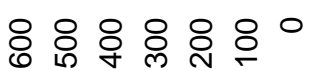

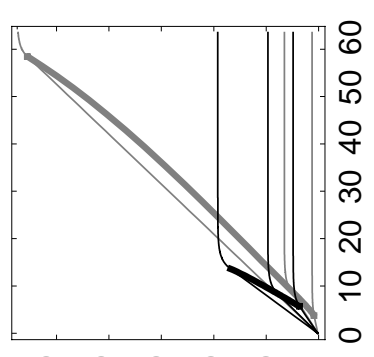

ঃ ঃ \& \& \& (u०) / 'บ1биәา

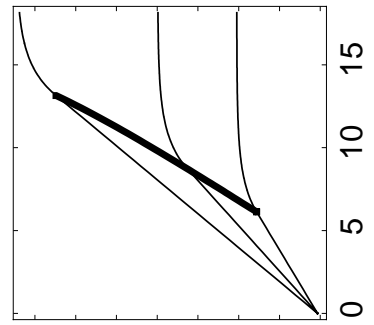

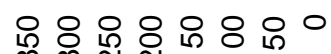

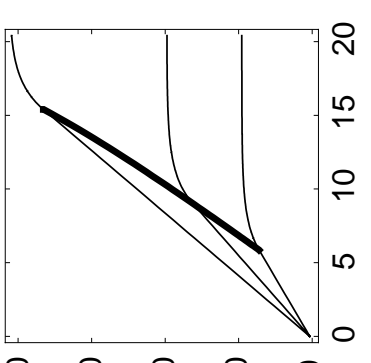

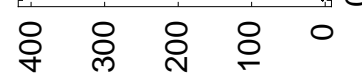

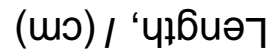

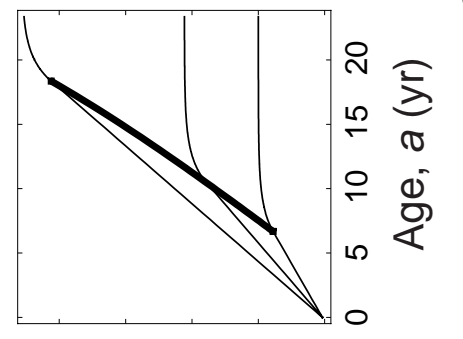

앙 \&

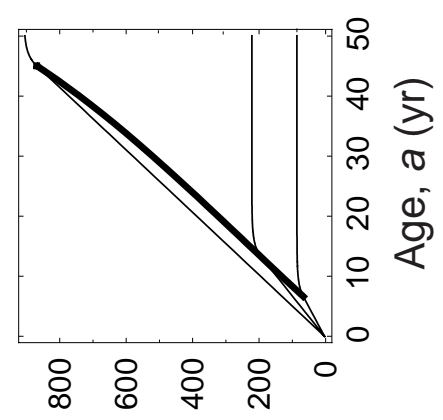

(mo) / 'บ1биәา

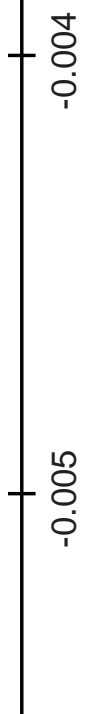

